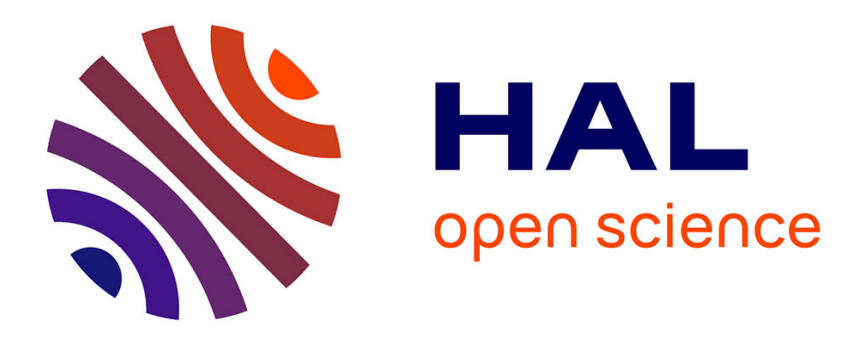

\title{
Spatial coherence of pipe vibrations induced by an internal turbulent flow
}

Laurent Maxit, Mahmoud Karimi, Oriol Guasch

\section{To cite this version:}

Laurent Maxit, Mahmoud Karimi, Oriol Guasch. Spatial coherence of pipe vibrations induced by an internal turbulent flow. Journal of Sound and Vibration, 2020, pp.115841. 10.1016/j.jsv.2020.115841 . hal-03025332

\section{HAL Id: hal-03025332 \\ https://hal.science/hal-03025332}

Submitted on 26 Nov 2020

HAL is a multi-disciplinary open access archive for the deposit and dissemination of scientific research documents, whether they are published or not. The documents may come from teaching and research institutions in France or abroad, or from public or private research centers.
L'archive ouverte pluridisciplinaire HAL, est destinée au dépôt et à la diffusion de documents scientifiques de niveau recherche, publiés ou non, émanant des établissements d'enseignement et de recherche français ou étrangers, des laboratoires publics ou privés. 


\title{
Spatial coherence of pipe vibrations induced by an internal turbulent flow
}

\author{
Laurent Maxit ${ }^{1}$, Mahmoud Karimi ${ }^{2}$, Oriol Guasch ${ }^{3}$, \\ 1. Univ Lyon, INSA-Lyon, Laboratoire Vibrations-Acoustique (LVA), 25 bis, av. Jean Capelle, F-69621, \\ Villeurbanne Cedex, France.
}

2. Centre for Audio, Acoustics and Vibration, University of Technology Sydney, Sydney, Australia

3. GTM - Grup de recerca en Tecnologies Mèdia, La Salle, Universitat Ramon Llull, C/ Quatre Camins 30, 08022 Barcelona, Catalonia, Spain

\begin{abstract}
:
Whereas the spatial coherence of wall pressure and vibratory fields induced by turbulent boundary layers (TBLs) on flat plates have been studied at extent, their equivalents for cylindrical structures still need further investigation. To that end, this work develops a semianalytical model which is valid for infinite cylindrical shells filled with a heavy fluid and excited by an internal TBL. The cylindrical shell can be also coupled to two ring stiffeners that account for the flanges generally used to connect a pipe to other portions of a circuit. The crossspectrum density (CSD) function of the shell radial accelerations is estimated from the system circumferential sensitivity functions and the CSD of the wall pressure field induced by the TBL. The spatial coherence of the pipe vibration field is therefore analysed for a pipe with and without flanges. This is of critical importance for applications such as non-intrusive techniques for detecting acoustic sources inside pipes, like beamforming using arrays of accelerometers. If the pipe conveys a flow, the beamforming efficiency can strongly deteriorate because of the background noise induced by the TBL, which pollutes the coherence signal between sensors. The effects that the spatial coherence could have on the beamforming results of a line of point sensors (accelerometers) and a ring of wire sensors (piezoelectric coiled wires) are also investigated in this paper.
\end{abstract}

Keywords: turbulent boundary layer, stochastic excitation, spatial coherence, cylindrical shell, ring stiffener, pipe, beamforming

\section{Introduction}

The prediction of the vibratory field of flat plates under air turbulent boundary layer (TBL) excitation has received a lot of attention (see e.g., [1] and [2]) and has been explored with analytical models of infinite and finite plates [3-5], numerical models [6-8] and experimental 
procedures $[9,10]$. Theoretical formulations are either expressed in the space-frequency or in the wavenumber-frequency domains [1]. For the former, the wall pressure field (WPF) induced by the TBL may be described e.g., by the renowned Corcos model [11] or the Mellen one [12], while for the second the Chase model is typically used [13].

Graham [14] reviewed existent TBL statistical models and investigated those suitable for transport aircraft. His study involved cylindrical structures like the ones we want to analyse in this work. One of the earliest works on TBL excitation of cylindrical shells was conducted by Norton and Bull [15]. The authors experimentally investigated the vibroacoustic response of a thin cylindrical pipe excited by a turbulent internal flow. In the plane wave propagation regime (low frequencies), the pipe wall peristaltic motion and resonant modes were shown to contribute to the structural response. At higher frequencies, acoustic coincidence effects led to strong excitation of supersonic pipe modes, which increase pipe wall vibrations and external noise radiation. A similar problem was addressed by Durant et al. [16], who resorted to a boundary integral formulation to perform numerical computations of the structural and acoustic responses of a thin cylindrical shell. The wall pressure excitation was described by the Corcos model with input parameters obtained from measurements of the cross spectral density (CSD) of the wall pressure fluctuations. Furthermore, a random vibration analysis of an axially compressed cylindrical shell in air excited by an external TBL was recently carried out by Li et al. [17]. The governing differential equations of the axially compressed cylindrical shell were derived and an eigenproblem was formulated using separation of variables. The CSD of the TBL was expanded as a Fourier series. Results for the shell responses were compared with those obtained from a modal decomposition method, showing good agreement.

Very recently, an analytical model of a fluid loaded cylindrical shell excited by an external turbulent flow field was presented by some of the authors of this work and colleagues in [18]. A wavenumber-point reciprocity principle $([19,20])$ was implemented to analytically determine the circumferential sensitivity functions of the shell, taking into account the fluid loading. The technique was used to investigate the physical mechanisms governing noise radiation by a cylindrical shell excited by a TBL. This was done by examining the contributions of individual circumferential modes to the acoustic spectra. The approach in [18] was extended to analyse noise radiation from a periodically stiffened shell under TBL excitation in [21]. The circumferential admittances that characterize the dynamic behaviour of the stiffeners were computed with a finite element model. Some bumps in the spectrum of the radiated pressure in the far field were observed, which were attributed to Bloch-Floquet waves propagating in the periodically stiffened shell. 
In the present paper, a similar theoretical approach to that in [18] and [21] is followed to analyse the spatial coherence of the vibrations of a fluid filled pipe, excited by an internal TBL. The pipe is modelled as an infinite cylindrical shell filled with water. Water is a heavy fluid (in comparison e.g., to air) and is therefore strongly coupled to the cylindrical shell vibrations. Such intense interaction is described in detail by the proposed theoretical vibroacoustic model. In addition, two configurations for the pipes are considered: a) a fluid filled infinite cylindrical shell and b) a fluid filled infinite cylindrical shell with two ring stiffeners.

The current work has significant applications for vibroacoustic beamforming techniques. In fact, it was mainly motivated by unexpected results from beamforming measurements in [22], to detect sodium-water reactions in the steam generator unit (SGU) of a liquid sodium fast reactor (SFR). Sodium/water reactions can lead to severe damage if appropriate action is not taken quickly. The presence of an internal TBL in the SGU can strongly pollute the beamforming outputs and limit their efficiency. The main issue here is to determine whether a weakly spatially correlated pressure field exerted by the TBL on the pipe wall may induce, or not, a strongly spatially correlated vibration field altering the beamforming results. To the best of the authors' knowledge, this question has not been dealt with before and has motivated the developments in this work.

Given the practical importance of the above problem in the complex framework of leak noise detection and propagation [23], it is worthwhile to explain it in some more detail. In recent years, a beamforming approach based on vibration measurements on the external hull of the SGU rather than on acoustic measurements [24-27] was studied in a collaboration between the French Alternative Energies and Atomic Energy Commission (CEA), and the Institut National des Sciences Appliquées (INSA) Lyon. The basis of the approach was developed in the framework of the PhD thesis of Moriot (2013). The numerical results by Moriot et al. [28] showed that vibroacoustic beamforming using a fixed linear array of accelerometers on the external surface of the cylindrical shell can be used to localize an acoustic monopole, as well as to increase the signal-to-noise ratio when the vibratory background noise is uncorrelated. To validate these results, an experiment on a laboratory mock-up was performed by Kassab et al. [22]. The experimental setup comprised a cylindrical pipe filled with a heavy fluid connected to a hydraulic circuit by two flanges (see Fig. 1). Water was used instead of sodium for practical reasons, which can be justified because water and sodium have similar densities and close bulk modulus values. A hydrophone in transmission mode inside the pipe generated the acoustic signal and the background noise was regulated changing the flow speed. Vibrations were 
measured with a line array of accelerometers with a spacing of $0.04 \mathrm{~m}$ (see Fig. 1). Special attention was paid to uncouple the test section from external mechanical sources (the pipe was fixed with rubber seals on a suspended slab) and from external fluid sources (using acoustic decoupling balloons). Upstream of the test section, a $4 \mathrm{~m}$ long pipe and a perforated plate flow conditioner were used to stabilize the turbulent flow, while a $1.5 \mathrm{~m}$ long pipe was set for discharging before the acoustic balloon. Despite all precautions, the background noise measurements showed a strong coherence between accelerometers at the considered flow speed. This behaviour was observed in the frequency range $[1 \mathrm{kHz}-3 \mathrm{kHz}]$ for a flow rate of $140 \mathrm{l} / \mathrm{s}$ $(4.5 \mathrm{~m} / \mathrm{s})$ as shown in Fig. 6 of [22]. Such a strong coherence significantly reduced the performance of the Bartlett beamforming approach, which relies on the assumption that the background noise is uncorrelated [29].

The strong coherence between sensor signals was unforeseen because the wall pressure field (WPF) induced by the TBL is supposed to be uniformly distributed on the pipe wall and it is also known to be weakly spatially correlated [11]. For a flow speed of $4.5 \mathrm{~m} / \mathrm{s}$, the WPF Corcos model [11] estimates a coherence length in the flow direction of $4.5 \mathrm{~cm}$ at $100 \mathrm{~Hz}, 1.5$ $\mathrm{cm}$ at $300 \mathrm{~Hz}$ and $0.2 \mathrm{~cm}$ at $2 \mathrm{kHz}$. Therefore, for frequencies in the vicinity of $300 \mathrm{~Hz}$ the sensor spacing is clearly greater than Corcos' coherence length. One would then presume very weak correlation between sensors beyond $300 \mathrm{~Hz}$, but this is not what was observed in Fig. 6 of Kassab et al. [22]. Yet there is an important difference between the experiments in [22] and previous works in literature. The latter mostly focus on the pressure induced by a TBL on a rigid wall, whereas the former focuses on the pipe shell vibrations induced by the TBL. As mentioned before, building a theoretical model to understand the spatial coherence of the pipe's vibration field is at the core of this work.

The paper is organized as follows. The theoretical models for the two pipe configurations are presented in Section 2. The CSD function of the radial acceleration between two axial positions on the pipe is given as a function of the WPF of the TBL, in the frequencywavenumber domain. An analytical expression of the circumferential sensitivity functions is derived for the fluid filled pipe and the circumferential admittance approach (CAA) is employed to model the stiffened pipe [30-32]. In Section 3, the proposed approach is used to analyse the auto and cross spectral densities (ASD and CSD) of a virtual line array of accelerometers, mimicking that in the experiment of Fig. 1 [22]. Comparisons between theory and experiments are also reported and the influence of various system parameters on the spatial coherence of the pipe's vibration field is analysed. In addition, the case of a virtual array of ring wire sensors is considered. The conclusions of the paper are finally drawn in Section 4, yet two appendices 
follow. The first one contains some technical details on the TBL and shell models, while the second one includes an additional comparison between the proposed model and the experimental results in [33].

\section{Theoretical formulation}

\subsection{Vibrational response of a cylindrical shell excited by an internal turbulent flow}

Let us consider an infinite cylindrical shell fully filled by a turbulent flow in the cylindrical coordinate system $(x, r, \theta)$, see Fig. 2. As mentioned in the Introduction, two configurations will be hereafter considered: a shell without stiffeners and a shell coupled to two ring stiffeners at the axial positions $x_{1}$ and $x_{2}$. The Flügge equations are used for the thin cylindrical shell and the coupling forces between shell and stiffeners are assumed to only take place in the radial direction. Given that the mean flow convection speed $U_{0}$ is small compared to the sound speed, the effects of convection on acoustic waves are neglected. This allows us to consider the Helmholtz equation for describing the acoustic pressure radiated inside the pipe. Moreover, the TBL exciting the inner wall of the shell is supposed to be spatially homogeneous, stationary and fully developed.

The CSD of the WPF, $\phi_{p p}$, is estimated from the TBL parameters and the Chase 1987 model [13], which is expressed in the wavenumber-frequency domain. Attention must be paid on how to define the space Fourier Transform for the problem at hand. In what follows, we employ a space Fourier transform about the $x$-axis and a Fourier series decomposition about the circumferential one (due to periodicity along the circumference). The Fourier transform, $\tilde{\tilde{f}}$, of a generic function $f$ depending on $x$ and $\theta$ is given by,

$$
f(x, \theta) \rightarrow \tilde{\tilde{f}}\left(k_{x}, n\right)=\frac{1}{2 \pi} \int_{-\infty}^{\infty} \int_{0}^{2 \pi} f(x, \theta) e^{-\mathrm{i}\left(k_{x} x+n \theta\right)} d \theta d x,
$$

where $k_{x}$ is called the axial wavenumber and $n$ is the circumferential order. Considering this definition of the Fourier transform, the CSD of the WPF in the $\left(k_{x}, n\right)$ domain due to the TBL, $\phi_{p p}^{t b l}$, can be related to the CSD of the WPF of Chase's model in [13], $S_{p p}^{t b l}$, through

$$
\phi_{p p}^{t b l}\left(k_{x}, n, \omega\right)=\frac{4 \pi^{2}}{R} S_{p p}^{t b l}\left(k_{x}, \frac{n}{R}, \omega\right) .
$$

For completeness, the Chase model considered in this paper is introduced in appendix A. 
From random vibrations theory, the CSD of the radial shell displacement between points of coordinates $(x, \theta)$ and $\left(x^{\prime}, \theta^{\prime}\right)$ on the shell, $S_{w w}$, can be written as [18, 21],

$$
S_{w w}\left(x, \theta, x^{\prime}, \theta^{\prime}, \omega\right)=2 \pi \sum_{n=-\infty}^{+\infty} \int_{-\infty}^{+\infty} \tilde{\tilde{H}}_{w}^{*}\left(x, \theta, k_{x}, n, \omega\right) \phi_{p p}^{t b l}\left(k_{x}, n, \omega\right) \tilde{\tilde{H}}_{w}\left(x^{\prime}, \theta^{\prime}, k_{x}, n, \omega\right) d k_{x} \text {, }
$$

where the circumferential sensitivity functions, $\tilde{\tilde{H}}_{w}$, can be expressed in the form

$$
\tilde{\tilde{H}}_{w}\left(x, \theta, k_{x}, n, \omega\right)=\frac{R}{2 \pi} \int_{-\infty}^{+\infty} \int_{0}^{2 \pi} H_{w}(\tilde{x}, \tilde{\theta}, x, \theta, \omega) e^{-\mathrm{i} k_{x} \tilde{x}} e^{-\mathrm{i} n \tilde{\theta}} d \tilde{x} d \tilde{\theta},
$$

making use of the reciprocity principle in $[18,20,21]$. In Eq. (4), $H_{w}(\tilde{x}, \tilde{\theta}, x, \theta, \omega)$ corresponds to the radial displacement of the shell at point $\widetilde{M}$ of coordinates $(\tilde{x}, \tilde{\theta})$ when the shell is excited by a unit radial force at point $M$ of coordinates $(x, \theta)$. Considering the definition of the Fourier transform in Eq. (1), the sensitivity functions $\tilde{\tilde{H}}_{w}$ of Eq. (4) correspond to the product of the shell radius and the Fourier transform of the radial displacements in the $\left(k_{x}, n\right)$ space, when the shell is excited by a unit radial point force at $M$. Equipped with this interpretation of the sensitivity functions, in next section we demonstrate how to estimate them for the fluid filled shell with and without ring stiffeners. The calculation process is based on the circumferential admittance approach (CAA) [30, 31].

Once $\phi_{p p}^{t b l}$ and $\tilde{\tilde{H}}_{w}$ are known, the numerical evaluation of Eq. (3) demands, in practice, truncating the wavenumber domain. For frequencies well above the hydrodynamic frequency, it is well known that the contribution of the convective peak is negligible to a good extent [34, 35]. Therefore, one can define an axial cut-off wavenumber $\bar{k}_{x}$ from the shell and fluid characteristics,

$$
\bar{k}_{x}=\kappa_{x} \max \left(k_{f}, k_{0}\right),
$$

where $k_{f}$ is the flexural wavenumber of a plate equivalent to the cylindrical shell (i.e. a flat plate having the same thickness and material properties as those of the shell), and $k_{0}$ is the acoustic wavenumber. The factor $\kappa_{x}$ is a safety coefficient typically taken to be $\kappa_{x}=2$. Similarly, one can define a cut-off circumferential order, $\bar{N}$, as follows

$$
\bar{N}=\operatorname{int}\left[\kappa_{N} R \max \left(k_{f}, k_{0}\right)\right]+1,
$$


where $\kappa_{N}$ is also a safety coefficient that can be set to $\kappa_{N}=1.5$. Considering the threshold values in Eq. (5) and Eq. (6), one can well estimate the CSD function of the radial displacement in Eq. (3) as,

$$
S_{w w}\left(x, \theta, x^{\prime}, \theta^{\prime}, \omega\right)=2 \pi \sum_{n=-\bar{N}}^{\bar{N}} \int_{-\bar{k}_{x}}^{\bar{k}_{x}} \tilde{\tilde{H}}_{w}^{*}\left(x, \theta, k_{x}, n, \omega\right) \phi_{p p}^{t b l}\left(k_{x}, n, \omega\right) \tilde{\tilde{H}}_{w}\left(x^{\prime}, \theta^{\prime}, k_{x}, n, \omega\right) d k_{x},
$$

and use a quadrature rule to compute the integral in the expression.

As illustrated in Fig. 3, the process for estimating the CSD of the radial displacement between points $(x, \theta)$ and $\left(x^{\prime}, \theta^{\prime}\right)$ consists of three main steps:

- First, computation of the sensitivity functions of the two points, $\tilde{H}_{w}\left(x, \theta, k_{x}, n, \omega\right)$ and $\tilde{\tilde{H}}_{w}\left(x^{\prime}, \theta^{\prime}, k_{x}, n, \omega\right)$ with the CAA approach (see next section).

- Second, evaluation of the Chase CSD of the WPF from Eq. (2) and Eq. (1A).

- Third and last, calculation of the CSD of the shell radial displacement using Eq. (7).

This process should be repeated for each angular frequency. Finally, let us mention that in this work results are presented for the CSD of the radial acceleration instead of displacement, which are related through $S_{\gamma}\left(x, \theta, x^{\prime}, \theta^{\prime}, \omega\right)=\omega^{4} S_{w w}\left(x, \theta, x^{\prime}, \theta^{\prime}, \omega\right)$. Moreover, given that the original Chase model for the TBL is a two-sided angular frequency spectrum, the CSD of Eq. (7) needs to be multiplied by a factor $4 \pi$ to convert it into a one-sided frequency spectrum.

\subsection{Estimation of the circumferential sensitivity functions}

In this section, we compute the radial displacement of the shell in the wavenumber domain $\left(k_{x}, n\right)$, when excited by a unit harmonic radial point force of angular frequency $\omega$ applied at point $M(x, \theta)$. Considering spectral displacements, $\tilde{\tilde{W}}_{F_{(x, \theta, \omega)}}\left(k_{x}, n\right)$, the sensitivity functions that appear in Eq. (7) can be obtained from $\tilde{\tilde{H}}_{w}\left(x, \theta, k_{x}, n, \omega\right)=R \tilde{\tilde{W}}_{F_{(x, \theta, \omega)}}\left(k_{x}, n\right)$. In what follows, they will be derived for the fluid filled cylindrical shell with and without ring stiffeners.

\subsubsection{Circumferential admittance approach (CAA)}

Fig. 4a shows an infinite cylindrical shell filled with a heavy fluid (water) and coupled to two ring stiffeners. For the modelling, the global system is initially partitioned into two subsystems 
namely, the shell and the ring stiffeners (see Fig. 4b). The CAA is then implemented to assemble them. The process succinctly consists in (see [30] for full details):

- First, as the considered system is axisymmetric (except for the excitation), the different spatial fields (displacement, acoustic pressure, force) generically denoted as $f$ can be represented by a Fourier series decomposition about the circumference,

$$
\begin{gathered}
\tilde{f}(n)=\frac{1}{2 \pi} \int_{0}^{2 \pi} f(\theta) e^{-\mathrm{i} n \theta} d \theta, \quad \forall n \in \mathbb{Z} \\
f(\theta)=\sum_{n=-\infty}^{\infty} \tilde{f}(n) e^{\mathrm{i} n \theta}
\end{gathered}
$$

- Second, for each circumferential order $n$ ( $n$ has been dropped from notation for simplicity), we define the circumferential admittances $\tilde{Y}_{i j}^{\alpha}$ of the fluid filled shell $(\alpha=1)$ and of the ring stiffeners $(\alpha=2)$, between the junctions $i \in\{1,2\}$ and $j \in\{1,2\}$ by,

$$
\tilde{Y}_{i j}^{\alpha}=\frac{\tilde{W}_{i j}^{\alpha}}{\tilde{F}_{i}^{\alpha}}, i \in\{1,2\}, j \in\{1,2\}
$$

where,

○ $\tilde{W}_{i j}^{\alpha}$ is the circumferential radial displacement at the junction $j$ due to the circumferential radial force applied at junction $i$;

○ $\tilde{F}_{i}^{\alpha}$ is the circumferential radial force applied at junction $i$ on the shell by the stiffeners $(\alpha=1)$ or the circumferential radial force applied on the stiffeners by the shell $(\alpha=2)$.

Moreover, for each subsystem $\alpha$, we define the circumferential free displacement at junction $i, \tilde{\bar{W}}_{i}^{\alpha}$, as the circumferential radial displacement induced by the external excitation at junction $i$, when all junctions are free.

- Finally, using the superposition principle for passive linear systems and considering radial displacement continuity and force equilibrium at the junctions, we obtain the matrix system $[30,31]$,

$$
\left[\tilde{\mathbf{Y}}^{1}+\tilde{\mathbf{Y}}^{2}\right] \tilde{\mathbf{F}}^{\mathbf{c}}=\tilde{\overline{\mathbf{W}}}^{2}-\tilde{\mathbf{W}}^{1}
$$

where $\tilde{\mathbf{Y}}^{\alpha}=\left[\begin{array}{cc}\tilde{Y}_{11}^{\alpha} & \tilde{Y}_{12}^{\alpha} \\ \tilde{Y}_{21}^{\alpha} & \tilde{Y}_{22}^{\alpha}\end{array}\right]$ is the circumferential admittance matrix of subsystem $\alpha$, $\tilde{\mathbf{F}}^{\mathbf{c}}=\left[\begin{array}{c}\tilde{F}_{1} \\ \tilde{F}_{2}\end{array}\right]$ is the circumferential coupling force vector containing the circumferential 
forces applied by the stiffeners on the shell when they are coupled together, and $\tilde{\overline{\mathbf{W}}}^{\alpha}=\left[\begin{array}{c}\tilde{\bar{W}}_{1}^{\alpha} \\ \tilde{\bar{W}}_{2}^{\alpha}\end{array}\right]$ is the circumferential free displacement vector of subsystem $\alpha$.

The solution of this system of equations provides the coupling forces that the stiffeners exert on the shell. Substituting them into the model for the infinite fluid filled shell we can estimate its vibratory field when coupled to the ring stiffeners. On the other hand, as no external excitation is applied to subsystem \#2, we get $\widetilde{W}_{i}^{2}=0$ in Eq. (11).

The above summarizes the basics of the CAA. In the next subsections, it is discussed how to estimate the circumferential admittances and the free displacements of the fluid filled shell, as well as those of the ring stiffeners. Furthermore, the shell radial displacements in the $\left(k_{x}, n\right)$ wavenumber space will be calculated as a response to the coupling forces and the external excitation.

\subsubsection{Spectral approach for estimating the shell circumferential admittances and free displacements}

The Flügge equations of motion of the cylindrical shell filled with water are given by ([36])

$$
\mathcal{L}(x, \theta)\left[\begin{array}{c}
U(x, \theta) \\
V(x, \theta) \\
W(x, \theta)
\end{array}\right]=\gamma\left\{\left[\begin{array}{c}
0 \\
0 \\
F_{\text {exc }}(x, \theta)
\end{array}\right]+\left[\begin{array}{c}
0 \\
0 \\
-p_{a}(x, \theta)
\end{array}\right]\right\},
$$

where $U, V$, and $W$ are respectively the longitudinal, tangential and radial displacements of the shell. $\mathcal{L}$ denotes the Flügge operator, $p_{a}$ is the pressure applied by the internal fluid on the shell and $\gamma=\left(1-v^{2}\right) R^{2} / E h$. Here, $h, E, v$ are the shell thickness, the shell Young modulus and the shell Poison ratio, respectively. $F_{e x c}(x, \theta)$ is the harmonic radial force distribution exerted with angular frequency $\omega$. Damping is introduced in the shell model through a complex Young modulus, $E^{*}=E(1+\mathrm{i} \eta)$, where $\eta$ is the structural damping loss factor. Applying the Fourier transform defined in Eq. (1) yields the shell equations of motion in the $\left(k_{x}, n\right)$ wavenumber domain, 


$$
\tilde{\tilde{\mathcal{L}}}\left(k_{x}, n\right)\left[\begin{array}{c}
\tilde{\tilde{U}}\left(k_{x}, n\right) \\
\tilde{\tilde{V}}\left(k_{x}, n\right) \\
\tilde{\tilde{W}}\left(k_{x}, n\right)
\end{array}\right]=\gamma\left\{\left[\begin{array}{c}
0 \\
0 \\
\tilde{\tilde{F}}_{\text {exc }}\left(k_{x}, n\right)
\end{array}\right]+\left[\begin{array}{c}
0 \\
0 \\
-\tilde{\tilde{p}}_{a}\left(k_{x}, n\right)
\end{array}\right]\right\} .
$$

The spectral Flügge matrix $\tilde{\tilde{\mathcal{L}}}\left(k_{x}, n\right)$ in Eq. (13) is provided in Appendix A.

On the other hand, the acoustic pressure satisfies the homogeneous Helmholtz equation [37],

$$
\Delta p_{a}(x, \theta, r)+\left(\frac{\omega}{c_{0}}\right)^{2} p_{a}(x, \theta, r)=0,
$$

and the kinematic condition at the cylindrical interface between the shell and the fluid medium,

$$
\frac{\partial p_{a}}{\partial r}(x, \theta, R)=\rho_{0} \omega^{2} W(x, \theta) .
$$

Here $\Delta$ stands for the Laplacian operator in the cylindrical coordinate system and $\rho_{0}, c_{0}$ respectively denote the fluid density and the sound speed. We introduce damping into the fluid through a complex celerity, $c_{0}{ }^{*}=c_{0}\left(1+\mathrm{i} \eta_{0}\right)$, where $\eta_{0}$ is the fluid damping loss factor. Applying the Fourier transform to Eqs. (14) and (15), the spectral radiated pressure $\tilde{\tilde{p}}_{a}$ can be related to the spectral radial displacement of the shell, $\tilde{\tilde{W}}$, through the spectral fluid impedance, $\tilde{\tilde{Z}}_{f}$, as [37-39],

$$
\begin{gathered}
\tilde{\tilde{p}}_{a}\left(k_{x}, n\right)=\tilde{\tilde{Z}}_{f}\left(k_{x}, n\right) \tilde{\tilde{W}}\left(k_{x}, n\right), \\
\tilde{\tilde{Z}}_{f}\left(k_{x}, n\right)=\frac{\rho_{0} \omega^{2}}{k_{r}} \frac{J_{n}\left(k_{r} R\right)}{J_{n}^{\prime}\left(k_{r} R\right)},
\end{gathered}
$$

where $J_{n}$ is the Bessel function of order $n$ and the prime denotes derivative with respect to the argument. The wavenumber $k_{r}$ is defined by

$$
k_{r}=\left\{\begin{array}{l}
\sqrt{k_{0}^{2}-k_{x}^{2}} \text { if }\left|k_{x}\right| \leq k_{0}, \\
-\mathrm{i} \sqrt{k_{x}^{2}-k_{0}^{2}} \text { otherwise. }
\end{array}\right.
$$

Substituting Eqs. (16) and (17) into Eq. (11), and inverting the matrix system, results in the shell spectral radial displacement, 


$$
\tilde{\tilde{W}}\left(k_{x}, n\right)=\frac{\gamma \tilde{\tilde{F}}_{e x c}\left(k_{x}, n\right)\left(\tilde{\tilde{Z}}_{U U}\left(k_{x}, n\right) \tilde{\tilde{Z}}_{V V}\left(k_{x}, n\right)-\left(\tilde{\tilde{Z}}_{U V}\left(k_{x}, n\right)\right)^{2}\right)}{\tilde{\tilde{\Delta}}\left(k_{x}, n\right)},
$$

where

$$
\begin{gathered}
\tilde{\tilde{\Delta}}=\tilde{\tilde{Z}}_{U W}\left(\tilde{\tilde{Z}}_{U V} \tilde{\tilde{Z}}_{V W}-\tilde{\tilde{Z}}_{U W} \tilde{\tilde{Z}}_{V V}\right)+\tilde{\tilde{Z}}_{V W}\left(\tilde{\tilde{Z}}_{U W} \tilde{\tilde{Z}}_{U V}-\tilde{\tilde{Z}}_{V W} \tilde{\tilde{Z}}_{U U}\right) \\
+\left(\tilde{\tilde{Z}}_{W W}-\gamma \tilde{\tilde{Z}}_{f}\right)\left(\tilde{\tilde{Z}}_{U U} \tilde{\tilde{Z}}_{V V}-\tilde{\tilde{Z}}_{U V}^{2}\right),
\end{gathered}
$$

and $\tilde{\tilde{Z}}_{m n}, \forall m, n=U, V, W$ are the elements of the spectral Flügge matrix $\tilde{\tilde{\mathcal{L}}}\left(k_{x}, n\right)$ in Appendix A.

Let us next contemplate two situations. In order to estimate the circumferential admittances of the fluid filled cylindrical shell, in the first one we consider the shell excited by a radial circumferential unit force $F$ at $x=0$, for each circumferential order $n$. The spectral radial force related to this excitation is $\tilde{\tilde{F}}_{\text {exc }}\left(k_{x}, n\right)=1$. The spectral radial displacement $\widetilde{W}$ can be calculated analytically using Eq. (19). The circumferential displacement $\widetilde{W}$ is then obtained from an inverse Fourier transform about $x$,

$$
\tilde{W}(x, n)=\frac{1}{2 \pi} \int_{-\infty}^{\infty} \tilde{\tilde{W}}\left(k_{x}, n\right) e^{i k_{x} x} d k_{x} .
$$

The integral is computed numerically after reducing the infinite integration limits to $\left[-\bar{k}_{x}, \bar{k}_{x}\right]$ , where $\bar{k}_{x}$ is the cut-off wavenumber defined in Eq. (5). As the considered system is translation invariant in the axial direction, the circumferential admittance of the shell between junctions $i$ and $j$ can be finally deduced from

$$
\tilde{Y}_{i j}^{1}(n)=\tilde{W}\left(x_{i}-x_{j}, n\right) .
$$

In the second situation, we use the same approach to compute the free circumferential radial displacements $\tilde{\bar{W}}_{i}^{1}, i \in\{1,2\}$, but now consider a unit point excitation, instead of a circumferential one, applied at point $M(x, \theta)$ and with spectrum,

$$
\tilde{\tilde{F}}_{\text {exc }}\left(k_{x}, n\right)=\frac{e^{-\mathrm{i}\left(k_{x} x+n \theta\right)}}{2 \pi} .
$$

Substituting Eq. (23) into Eq. (19) and inverse Fourier transforming through Eq. (21), the radial circumferential displacement of the shell can be obtained. The free displacement at the junction $i$ is then given by $\tilde{\bar{W}}_{i}^{1}(n)=\tilde{W}\left(x_{i}, n\right), i \in\{1,2\}$. 


\subsubsection{Circumferential admittances of the ring stiffener}

In what follows we suppose that the two ring stiffeners and the shell are made of the same material. A model of a circular rod and of rectangular cross-section [40] simulates the stiffeners' dynamic behaviour. We define $l_{r}$ and $h_{r}$ as the width and height of the cross section, respectively. Neglecting inertia and rotary effect, the radial circumferential admittance, $\tilde{Y}_{r}$ can be estimated as [40],

$$
\tilde{Y}_{r}(n)=\frac{R^{2}\left[\Omega_{r}{ }^{2}-n^{2}\left(1+\beta_{r}{ }^{2}\right)\right]}{E l_{r} h_{r}\left[\left(1-\Omega_{r}{ }^{2}+\beta_{r}{ }^{2} n^{4}\right)\left(\Omega_{r}{ }^{2}-n^{2}\left(1+\beta_{r}{ }^{2}\right)\right)+n^{2}\left(1+\beta_{r}{ }^{2} n^{2}\right)^{2}\right]}
$$

where $\beta_{r}=\frac{h_{r}{ }^{2}}{12 R^{2}}$ and $\Omega_{r}=R \omega \sqrt{\frac{\rho}{E}}$.

Given that the two stiffeners are not physically coupled to each other, $\tilde{Y}_{12}^{2}=\tilde{Y}_{21}^{2}=0$, and because they are identical we get $\tilde{Y}_{11}^{2}=\tilde{Y}_{22}^{2}=\tilde{Y}_{r}$.

\subsubsection{Displacements of the shell coupled to the ring stiffener}

Once the shell and stiffener admittances have been obtained, as well as the free displacements induced by the external excitation, it becomes possible to compute the coupling forces with Eq. (11). The forces applied on the fluid loaded cylindrical shell are the external unit radial point force applied at point $M$ of coordinates $(x, \theta)$ and the radial circumferential forces applied by the ring stiffeners. These forces should be reinjected in the spectral model of the fluid filled shell described in Section 2.2.2 to determine the radial displacement of the fluid loaded shell, when coupled to the two ring stiffeners. The spectral radial forces are given by,

$$
\tilde{\tilde{F}}_{e x c}\left(k_{x}, n\right)=\frac{e^{-\mathrm{i}\left(k_{x} x+n \theta\right)}}{2 \pi}+\tilde{F}_{1} e^{-\mathrm{i} k_{x} x_{1}}+\tilde{F}_{2} e^{-\mathrm{i} k_{x} x_{2}} .
$$

but Eq. (25) is then substituted into Eq. (19) to obtain the spectral radial displacement of the fluid filled shell coupled to the stiffeners, $\tilde{\tilde{W}}_{F_{(x, \theta, \omega)}}\left(k_{x}, n\right)$. The sensitivity functions of the considered system that appear in Eq. (7) are finally computed taking into account that $\tilde{\tilde{H}}_{w}\left(x, \theta, k_{x}, n, \omega\right)=R \tilde{\tilde{W}}_{F_{(x, \theta, \omega)}}\left(k_{x}, n\right)$. 


\section{Analysis of the numerical results}

This section focuses on using the proposed semi-analytical model for the analysis of the shell vibratory field induced by the internal TBL. Special emphasis is paid to spatial coherence. The investigations are carried out on a configuration that mimics the test pipe of the experimental set-up in Fig. 1, also considered in the experimental study [22]. For characterizing the shell vibration field, we emulate two types of virtual array sensors:

1. A virtual linear array of standard "point" accelerometers representing the situation depicted in Fig. 1

2. A virtual ring array simulating piezoelectric wires wound around the shell an integral number of times ([41]). Only the component $n=0$ of the radial acceleration needs to be evaluated in this case.

Section 3 is organized as follows. The test cases to be analysed are described in Subsection 3.1, while the dynamic characteristics of the shell and ring stiffeners are presented in Subsection 3.2. Results for the ASD of the radial accelerations induced by the TBL are discussed in Subsection 3.3 and those of the normalized CSD in Subsection 3.4. A comparison with the experimental results in [22] is presented in Subsection 3.5, followed by an analysis of the system parameter influence on the spatial coherence of the vibration field in Subsection 3.6. Finally, Subsection 3.7 concentrates on the ASD and normalized CSD for the component $n=0$ of the radial acceleration.

\subsection{Description of the test case and modelling approach}

The laboratory experiment in [22] that will be mimicked hereafter consisted of a test section pipe of length $3.1 \mathrm{~m}, 0.219 \mathrm{~m}$ in diameter and a thickness of $0.008 \mathrm{~m}$. Upstream of the test section, a $4 \mathrm{~m}$ long pipe was used to stabilize the turbulent flow, while a $1.5 \mathrm{~m}$ long pipe was attached afterwards for discharge. These two pipes had the same dimensions as the test pipe and were connected to it by means of two flanges. Both the pipe and the flanges were made of stainless steel, whose material properties are taken as $\rho=7800 \mathrm{~kg} / \mathrm{m}^{3}, E=185 \mathrm{GPa}, v=0.33$ ,$\eta=0.001$. The water flow rate was $140 \mathrm{l} / \mathrm{s}$ which corresponds to a flow speed of $4.5 \mathrm{~m} / \mathrm{s}$. The density, speed of sound and the loss factor for water are $\rho_{0}=1000 \mathrm{~kg} / \mathrm{m}^{3}, c_{0}=1500 \mathrm{~m} / \mathrm{s}$ and $\eta_{0}=0.001$.

To model the above set-up, the stabilizing and discharge pipes are represented as infinite cylindrical shells. The flanges are simulated by ring stiffeners of rectangular cross-section 
$0.04 \mathrm{~m}$ (width) $\times 0.06 \mathrm{~m}$ (height) and are placed at the axial positions $x_{1}=0 \mathrm{~m}$ and $x_{2}=3.1 \mathrm{~m}$. Concerning the TBL excitation, the wall pressure field is represented by the Chase model [13]. The TBL thickness is assumed equal to the shell radius, whereas the convective and friction velocity are respectively taken as $U_{c}=0.7 U_{0}$, and $U_{\tau}=0.032 U_{0}$. The frequency band of interest has been fixed to $[100 \mathrm{~Hz}-2000 \mathrm{~Hz}]$ with a frequency resolution of $4 \mathrm{~Hz}$.

\subsection{Shell and ring stiffener characteristics}

Before analysing the vibratory field of the shell in response to the TBL excitation, it is worth investigating the dynamics of the shell and stiffeners.

We start with the circumferential admittances needed for evaluating the coupling force between the shell and the ring stiffeners, in Eq. (11). The magnitudes of the circumferential admittances of the ring stiffeners for a large frequency range (i.e. [1 Hz $-10 \mathrm{kHz}]$ ) and for the circumferential orders $n$ from 0 to 3, are plotted in Fig. 5a. These values have been calculated with Eq. (24). The peaks observed in the figure can be attributed to the modes of the stiffener and take place at resonance frequencies $7352 \mathrm{~Hz}, 0 \mathrm{~Hz}, 3158 \mathrm{~Hz}$ and $8800 \mathrm{~Hz}$ for $n=0,1,2,3$, respectively. The beam mode associated to the circumferential order $n=1$ corresponds to a rigid body mode that explains the "peak" at $0 \mathrm{~Hz}$. The ring mode associated to the circumferential order $n=0$ exhibits a resonance frequency higher than that for $n=2$ due to the effect of curvature of the ring stiffener. Withal, note in Fig. $5 b$ that in the frequency band of interest $[100 \mathrm{~Hz}-2000$ $\mathrm{Hz}]$ the stiffeners do not show any resonance. If one compares these curves with those in Fig. $5 \mathrm{c}$ for the admittances of the fluid filled shell, the following is observed. First, and for the same reasons as for the stiffeners, the shell exhibits "resonances" outside the frequency band of interest for circumferential orders $n=0$ and $n=1(6623 \mathrm{~Hz}$ and $0 \mathrm{~Hz}$, respectively). Yet and opposed to the stiffeners, one can observe peaks belonging to the range $[100 \mathrm{~Hz}-2000 \mathrm{~Hz}]$ for circumferential orders $n=2$ and $n=3$, at $360 \mathrm{~Hz}$ and $1072 \mathrm{~Hz}$ respectively. Strictly speaking, one cannot associate these frequency peaks to modes of the fluid filled shell because the shells are unbounded. These frequencies are usually referred in the literature as the cut-on frequency of each circumferential order, or each circumferential mode, see [38, 39]. Below this specific frequency, the waves propagating in the axial direction are evanescent while they become propagative above it.

Comparing the values of the admittances of the ring stiffeners in Fig. 5b with those of the shell in Fig. 5c reveals which of the two subsystems is the stiffest one. In particular, one notices that for the circumferential orders $n=2$ and $n=3$, the shell admittances are more than one order of magnitude greater than those of the stiffener, regardless of frequency. For these 
circumferential orders, the stiffener is therefore much more rigid than the shell. Consequently, when the two subsystems are coupled, the stiffeners will have the tendency to confine the shell vibrations. In contrast, for the circumferential order $n=1$ the shell admittances are smaller than those of the stiffener at low frequencies. For the latter, the influence of the ring stiffeners on the shell vibrations is therefore expected to be rather limited.

The coupling forces between shell and stiffeners are calculated inserting the admittances in Eq. (11). Plugging their values into the spectral force expression Eq. (25), we can estimate the spectrum of the shell radial displacements from Eq. (19). To analyse them, as well as the waves propagating in the fluid in the axial direction, it suffices computing the spectral displacements for unit spectral forces (i.e. taking $\tilde{\tilde{F}}\left(k_{x}, n\right)=1$ in Eq. (19)). This is equivalent to consider the shell is excited by a point force at $(0,0)$, with an amplitude of $2 \pi$. Fig. 6 shows the displacements depending on frequency and the axial wavelength $\lambda_{x}=2 \pi / k_{x}$, for the first four circumferential orders. One can observe some trajectories that can be associated to operational dispersion curves, which identify propagative waves. These curves differ from classical dispersion curves (obtained by searching the roots of the denominator $\tilde{\tilde{\Delta}}$ of Eq. (19)), by the fact that they depend on the excitation. The levels of the trajectories give us an indication of the shell sensitivity to the input excitation. In the present case of a radial excitation, one can observe in Fig. 6 that the shell response for the circumferential order $n=0$ is very small in comparison with the other orders. On the contrary, significant displacement is produced for the circumferential order $n=1$ in the entire frequency range. The shell also strongly reacts for circumferential orders $n=2$ and $n=3$, above their respective cut-on frequencies.

As the turbulent flow excites the shell radially, one would expect the above characteristics of the circumferential orders to play a significant role in determining the vibratory field of the shell when excited by a TBL. Moreover, the trajectories in Fig. 6 are an indication of the wavelength of the propagative waves in the fluid filled shell. For the circumferential order $n=0$, in Fig. 6a we have superposed the acoustic wavelength as well as the longitudinal and flexural wavelengths of an equivalent plate with the same thickness and material properties as those of the shell. From the proximity of the superimposed trajectories with the maximum spectral displacement curves, one could associate the first trajectory from the left to dominant acoustic behaviour of the fluid, while the second can be identified with compressional-extensional motions of the shell (which lead to radial displacement through the Poisson effect). For higher 
circumferential orders, the trajectories are the results of the strong interactions between the shell and fluid motions and the figures are more difficult to interpret.

\subsection{Auto-spectrum density for the virtual line array of accelerometers}

To analyse the vibration field of the pipe test section, we next consider the first virtual array configuration introduced at the beginning of Section 3. The array is composed of 25 virtual accelerometers fixed on the cylindrical generator at $\theta=0$ (similar to the experiment in Fig. 1). The spacing between the sensors is $\Delta x=0.04 \mathrm{~m}$ and the first accelerometer is positioned at $0.1 \mathrm{~m}$ from the upstream flange of the flow. The coordinates of the $m$-th accelerometer are therefore $\left(x_{m}, \theta_{m}\right)=(0.06+0.04 m, 0)$ for $m \in\{1, \ldots, 25\}$. As said at the end of Section 2.1, the acceleration ASD or CSD, $S_{\gamma \gamma}$, is used hereafter, which is related to displacement ASD or CSD, $S_{w w}$, obtained from Eq. (7), by $S_{\gamma \gamma}=\omega^{4} S_{w w}$.

Fig. 7 presents the mean value of the ASD of the radial accelerations measured by the 25 virtual accelerometers. The ASD has been computed with the flow conditions presented in Subsection 3.1. Results are given for the fluid filled shell without and with two ring stiffeners. For the former (Fig. 7a), one can notice two significant peaks, at $360 \mathrm{~Hz}$ and $1072 \mathrm{~Hz}$, which correspond to the cut-on frequencies of the shell admittance introduced in the previous subsection for $n=2$ and $n=3$. The individual contributions of each circumferential order are also plotted in the figure confirming the prominence of $n=2$ and $n=3$ for most of the ASD, except for frequencies below $360 \mathrm{~Hz}$, where the circumferential order $n=1$ becomes dominant. It should be noted that the contribution of $n=0$ is negligible, with values lower than $-140 \mathrm{~dB}$, so it is not plotted in the figure.

Concerning the case of the shell coupled with the two ring stiffeners in Fig. $7 \mathrm{~b}$ numerous additional peaks appear as compared to Fig. 7a, which can be attributed to circumferential orders $n=2$ and $n=3$, none of them being associated to $n=1$. This can be explained by the fact that for $n=2$ and $n=3$, the stiffeners are more rigid than the shell. Therefore, propagative waves in the fluid filled shell experience significant reflection at the stiffeners. The interferences between the outward propagating waves and the reflected ones lead to resonances at some frequencies. In the following, let us term the resulting waves as "pseudo axial modes", as they consist in axial standing waves built from partial wave reflexion at the stiffeners. Pseudo axial waves do not occur for $n=1$ because the shell is more rigid than the stiffener for this circumferential order. Consequently, propagative waves barely perceive the stiffeners and 
almost no reflection occurs. The frequency related to the pseudo axial modes can be approximated from the knowledge of the propagative axial wavelength given in Fig. 6 for each circumferential wavenumber. Indeed, it is well-known that resonances happen when the interference between the outward propagating waves and the reflected ones are constructive, which leads to standing waves exhibiting nodes of vibration separated by half a wavelength. For instance, for a basic system as a simply supported beam, resonances occur when the beam length is exactly a multiple of half-wavelength. In order to verify that the pseudo axial modes follow a similar law for each circumferential order, one can identify the frequencies, $f_{m}^{\text {approx }}$ for which the distance between the two stiffeners correspond to a multiple $m$ of half the axial propagative wavelength, $\lambda_{x}$, given in Fig. 6:

$$
f_{m}^{\text {approx }} \in \mathbb{R}^{+} / L_{x}=m \frac{\lambda_{x}\left(f_{m}^{\text {approx }}\right)}{2},
$$

where $L_{x}=x_{2}-x_{1}$ corresponds to the length of the test section.

Table 1 compares the resonant frequencies for the circumferential order $n=2$ obtained using the relation (26) and from the experiment [22], with those predicted from the frequency values of the peaks observed in Fig. 7. A good agreement is observed among results. One can check that the progression of the resonant frequencies is well represented by the approximate law. The frequencies of the additional peaks in Fig. $7 \mathrm{~b}$ as compared to those in Fig. $7 \mathrm{a}$ can be well associated with constructive interferences between the outward propagating waves and the reflected ones (i.e., the pseudo axial modes). It should be noted that we refuse to use the term axial modes because normal modes are only defined for bounded systems. In the present case, both the shell and the cylindrical fluid cavity remain unbounded.

Table 1. Comparison of the resonant frequencies related to the circumferential order $n=2$ in function of the axial modal order, $m: f_{m}^{\text {approx }}$, approximate frequency verifying the relation (26); $f_{m}^{\text {frame }}$, frequency of the $m^{\text {th }}$ resonant peak observed in Fig. 7 for the shell coupled to the two ring stiffeners; $f_{m}^{\text {exp }}$, frequency of the $m^{\text {th }}$ resonant peak observed above $360 \mathrm{~Hz}$ in the experiment acceleration spectra for a hammer excitation [22].

\begin{tabular}{|c|c|c|c|c|c|c|c|c|c|c|}
\hline$m$ & 1 & 2 & 3 & 4 & 5 & 6 & 7 & 8 & 9 & 10 \\
\hline$f_{m}^{\text {approx }}(\mathrm{Hz})$ & 360 & 369 & 392 & 442 & 522 & 632 & 767 & 920 & 1068 & 1265 \\
\hline$f_{m}^{\text {frame }}(\mathrm{Hz})$ & 360 & 372 & 400 & 456 & 544 & 660 & 800 & 956 & 1124 & 1304 \\
\hline$f_{m}^{\text {exp }}(\mathrm{Hz})$ & 362 & 374 & 400 & 450 & 540 & 656 & 780 & 934 & - & - \\
\hline
\end{tabular}


The formation of pseudo axial modes is confirmed in Fig. 8, where we plot the distributions of the ASD of the acceleration as a function of the accelerometer position and frequency. The pattern in the figure at resonance frequencies exhibits some bumps and nodes. The distances between two nodes can be related to half the wavelength of the propagative waves detected in Fig. 6. For instance, one can observe from Fig. 8 that this distance is close to 0.48 $\mathrm{m}$ at $660 \mathrm{~Hz}$ and $0.36 \mathrm{~m}$ at $956 \mathrm{~Hz}$, and we found respective wavelengths of $0.98 \mathrm{~m}$ and $0.74 \mathrm{~m}$ for these two frequencies and $n=2$ in Fig. 6c. Similarly, at higher frequencies, one finds that the distance between two nodes is $0.4 \mathrm{~m}$ at $1258 \mathrm{~Hz}$ and $0.24 \mathrm{~m}$ at $1828 \mathrm{~Hz}$, which corresponds well to half the wavelength for $n=3$ in Fig. $6 \mathrm{~d}$. This confirms that the pseudo axial modes are built from constructive interferences between the propagating waves and the reflected ones at the ring stiffeners.

The ASD of the radial vibration of the shell excited by the TBL has been evaluated using Eq. (7). It depends on the product of the circumferential sensitivity functions of the shell and the CSD of the WPF. The latter characterises the TBL excitation that can be associated with a set of uncorrelated wall plane waves $[8,34,42]$, whereas the former are space transfer functions characterising the vibro-acoustic response of the fluid filled shell (with or without stiffeners) to unit wall plane wave (WPW) excitation. In the present study, the frequency band of interest is well above the hydrodynamic coincidence frequency. Given that the CSD of the WPF is relatively flat in the sub-convective domain [1], the uncorrelated WPWs representing the TBL excitation have similar amplitudes. The response of the shell to the TBL is then mainly controlled by the response of the shell to WPW excitation. The analysis of the sensitivity functions can help us to better understand how the TBL excites the shell. These are plotted in Fig. 9 as a function of the positive axial wavenumber for $n=2$ and at $660 \mathrm{~Hz}$, which corresponds to the frequency of the pseudo axial mode $(m, n)=(6,2)$. The results are given at the position of sensors \#6 and \#13 for the shell with two ring stiffeners: the first position corresponds to an antinode of the pseudo axial mode whereas the second one corresponds to a node. For comparison, the result for the shell without stiffeners is also given (independently of the sensor position). One can observe a peak indicating that the shell (with or without stiffeners) reacts mainly to the WPW that has a wavenumber close to $6.4 \mathrm{rad} / \mathrm{m}$, i.e., to a wavenumber similar to that of the propagative wave for $n=2$ (as $\lambda_{x}=0.98 \mathrm{~m}$ at $660 \mathrm{~Hz}$, see Fig. 6c). The amplitude of the peak is more important for position \#6 than for position \#13, the difference being of $\sim 12$ $\mathrm{dB}$. This explains why the ASD of the radial acceleration of the shell excited by the TBL for sensor \#6 is also about $12 \mathrm{~dB}$ higher than that for sensor \#13 at $660 \mathrm{~Hz}$. Fig. 10 shows the 
amplitude of the sensitivity function for all sensors, confirming our previous analysis on two sensors. It is observed that regardless of the sensor position, two peaks occur at wavenumbers $\pm 6.4 \mathrm{rad} / \mathrm{m}$. Moreover, the amplitude of these peaks for the shell without stiffeners is $10 \mathrm{~dB}$ lower than that of the shell with ring stiffeners for almost all sensors. This explains again the $10 \mathrm{~dB}$ difference in the acceleration ASD between the two shell models at $660 \mathrm{~Hz}$, which was observed in Fig. 7. The phases shown in Figs. 10c-d will be discussed in the following section.

\subsection{Normalized cross-spectrum density for the virtual line array of accelerometers}

Having studied the ASD of the shell radial accelerations, let us next focus on the spatial coherence of the vibratory field, which was the main motivation of the present work. As mentioned in the Introduction, the coherence length of the TBL in the axial direction for the considered flow speed is smaller than the sensor spacing $(0.04 \mathrm{~m})$, at frequencies equal or greater than $300 \mathrm{~Hz}$. Hence, the wall pressures induced by the TBL and measured by hydrophones next to the accelerometers should be weakly correlated. To illustrate this point, the normalized CSD of the wall pressure measured by a virtual array of 7 hydrophones is presented in Fig. 11. The hydrophones are positioned at coordinates $\left(x_{m}, \theta_{m}\right)=(0.06+0.04 m, 0), \forall m \in\{1, \ldots, 7\}$. The normalized CSD of the wall pressure between the $i$-th and $j$-th hydrophones can be calculated as,

$$
\Gamma_{i j}^{p}(\omega)=\frac{\left|S_{p p}\left(x_{i}-x_{j}, \theta_{i}-\theta_{j}, \omega\right)\right|}{S_{p p}(0,0, \omega)},
$$

where $S_{p p}$ is the space-frequency spectrum of the wall pressure field evaluated from the numerical inverse Fourier transform of the wavenumber-frequency spectrum of the Chase model [13] (see appendix A). One can clearly observe in Fig. 11 that the normalized CSD of the wall pressure is very low in general. For two adjacent hydrophones, the normalized CSD is less than 0.1 for frequencies above $350 \mathrm{~Hz}$. When the spacing between two hydrophones is equal or greater than $12 \mathrm{~cm}$, the normalized CSD is less than 0.1 for all frequencies in Fig. 11.

The question that we want to next address is how correlated the vibratory field is when the shell is excited by a uniformly distributed and weakly spatially correlated WPF? To answer this query, we introduce the normalized CSD of the radial acceleration between the $i$-th and $j$ th accelerometers of our virtual array as,

$$
\Gamma_{i j}^{\gamma}(\omega)=\frac{\left|S_{\gamma \gamma}\left(x_{i}, \theta_{i}, x_{j}, \theta_{j}, \omega\right)\right|}{\sqrt{S_{\gamma}\left(x_{i}, \theta_{i}, x_{i}, \theta_{i}, \omega\right) S_{\gamma}\left(x_{j}, \theta_{j}, x_{j}, \theta_{j}, \omega\right)}},
$$


where $S_{\gamma}$ is the CSD of the radial acceleration obtained from Eq. (7) and $S_{\gamma}=\omega^{4} S_{w w}$. The normalized CSD is calculated for all hydrophone pairs in the array and plotted in Fig. 12, for the cases of the fluid filled shell with and without stiffeners. The figure presents results for three different frequencies, namely $1072 \mathrm{~Hz}$ that corresponds to the cut-on frequency of $n=3,1200$ $\mathrm{Hz}$ that matches with a pseudo axial mode related to $n=3$ (for the stiffened shell) and $1228 \mathrm{~Hz}$ that falls in the "non-resonant" domain of the stiffened shell.

Clearly, one can notice that for the two shell configurations, the normalized CSD is close to one for all hydrophone pairs at the cut-on frequency of $n=3$ (see Figs. 12a and 12d). At this frequency, the shell vibration field has a strong spatial correlation despite the excitation does not. For the frequency corresponding to a pseudo axial mode (Figs. 12b and 12e), the CSD patterns associated to the non-stiffened and stiffened shells are very different yet values close to one are reached in both cases. For the stiffened shell in Fig. 12e, only the two accelerometers \#10 and \#21 exhibit very low correlation with other sensors. The reason for this behaviour is that these accelerometers are axially positionned at $x_{10}=0.46 \mathrm{~m}$ and $x_{21}=0.9 \mathrm{~m}$, which coincide with the nodes of the pseudo axial mode, as observed in Fig. 8. As regards the patterns for the third frequency in Figs. 12c and 12f for the non-stiffened and stiffened shells, they are very similar because in this case the frequency does not coincide with any resonance of the stiffened shell. It can be observed in the figure that several couples of accelerometers present significant values of normalized CSD.

To strengthen the spatial coherence analysis, in Fig. 13 we depict the normalized CSD of the radial acceleration between accelerometer \#1 and all other accelerometers, as a function of frequency. The figure clearly reveals that the vibratory field of the shell displays significant spatial correlation for the whole frequency range, although the excitation is almost spatially uncorrelated (as shown in Fig. 11). However, not all signals are strongly correlated. The white patterns in Fig. 13 stand for strong coherence regions which can be related to the vibroacoustic characteristics of the shell. For the fluid filled shell without the stiffeners, the cut-on frequencies at $360 \mathrm{~Hz}$ and $1072 \mathrm{~Hz}$ lead to unit spatial coherence of accelerometer \#1 with all other sensors, but the situation is very different for the remaining frequencies. The distance between the curves of two low correlated accelerometers (corresponding to the black zone in Fig. 11) can be related to the wavelength of the propagative vibro-acoustic waves shown in Fig. 6. For instance, at the two frequencies $660 \mathrm{~Hz}$ and $956 \mathrm{~Hz}$ analysed in Subsection 3.3, this distance is respectively $0.48 \mathrm{~m}$ and $0.36 \mathrm{~m}$, which corresponds to half wavelength of the propagative waves for $n=2$ in 
Fig. 6c. Comparing Fig. 8 and Fig. 13b for the stiffened shell, it becomes possible to relate the patterns of strong coherence to pseudo axial modes.

The analysis of the sensitivity functions shown in Fig. 10 at $660 \mathrm{~Hz}$ can enlighten the underlying phenomenon involved in these strong coherences. Indeed, we have already observed in section 3.3 that efficient WPW characterized by wavenumbers close to the propagative wavenumbers (i.e. $6.4 \mathrm{rad} / \mathrm{m}$ for the case of Fig. 10) and its opposite are the main contributors to the vibratory response of the shell when it is excited by the TBL. This corresponds to the peaks observed in the sensitivity functions in Figs. 10 a-b. On the other hand, Figs. 10c-d show the phase differences of the sensitivity functions between sensor \#1 and all the other phases of the shell response to the WPW, as a function of sensor position. For the shell without stiffeners, it is seen in Fig. 10c that the phase difference varies significantly with position, which is the result of wave propagation. Focusing on the phase difference related to the two peaks, it is noticed that this one depends on the sensor position. For instance, if we consider sensor \#6, the phase difference with sensor \#1 is $1.2 \mathrm{rad}$ for the peak at $6.4 \mathrm{rad} / \mathrm{m}$ and $-1.2 \mathrm{rad}$ for the one at $-6.4 \mathrm{rad} / \mathrm{m}$, whereas for sensor \#13 the phase differences are respectively $3.1 \mathrm{rad}$ and $-3.1 \mathrm{rad}$. The phase differences between the two peaks for sensors \#6 and \#13 are respectively 2.4 rad and 6.2 rad. Hence, the contributions of the two peaks partially vanish for sensor \#6 whereas they add up for sensor \#13 (as $6.2 \approx 2 \pi$ ). Therefore, the coherence between sensors \#1 and \#6 is weak at this frequency as it can be seen from Fig. 13a, while it is close to one between sensors $\# 1$ and \#13.

A similar analysis can be carried out for the shell with two ring stiffeners. In this case, one can notice in Fig. 10d that the phase shift with sensor \#1 does not vary significantly with the sensor position as much as with wavenumber, with the exception of sensor locations close to the node of the pseudo axial mode $(m, n)=(6,2)$ (i.e. close to $0.6 \mathrm{~m})$. The phase shift between the two peaks is then almost $0(2 \pi)$. Their contributions are constructive which results in a strong coherence between sensor \#1 and all other ones (with the cited exception of those close to node $(m, n)=(6,2))$.

To conclude this section, we can assert that, for the considered cases, it is certainly feasible that a weakly spatially correlated TBL gives place to a strongly spatially correlated vibration field on a fluid filled shell. 


\subsection{Comparison with experimental results}

This section presents a comparison between the numerical results obtained using the proposed model and the experimental results obtained from the laboratory mock-up in [22]. The experimental conditions have been briefly presented in the Introduction and for a full description the reader is referred to [22]. As detailed in section 3.1, the physical and geometrical parameters used in the numerical simulations are the same ones used in the experiments of [22].

Fig. 14a shows the radial displacement response measured with a $2 \mathrm{~Hz}$ frequency resolution when the system is excited by a unit radial point force with a hammer at $x=0.105 \mathrm{~m}$. This forced response under a controlled excitation contain the pseudo-axial modes. The patterns observed in Fig. 14a can be attributed to the spatial shape of the pseudo-axial modes, which present significant similarities to the ones already revealed in Fig. 8. Moreover, some peaks can be detected in the experimental spectra between $200 \mathrm{~Hz}$ and $1000 \mathrm{~Hz}$. According to the simulations, these peaks are associated with the pseudo-axial modes corresponding to the circumferential order $n=2$. These experimentally obtained frequencies, $f_{m}^{\exp }$, are compared to the ones identified in the numerical spectra, $f_{m}^{\text {frame }}$, in Table 1. A good agreement for the first 6 modes as well as for the distribution of higher modes is achieved. The discrepancies in the higher modes may have different origin and could be due to the fact that (a) frequencyindependent standard values for the mechanical properties of the shell and ring stiffener material have been considered in the simulation; (b) the coupling between the shell and the ring stiffeners is modelled only in the radial direction; (c) the pipe in the experiment is finite whereas it is infinite in the simulation with some supports used to fix it on the suspended slab. Despite of such issues, however, we believe the comparison demonstrates that the developed model well represents the main vibratory behaviour of the experimental mock-up.

On the other hand, Fig. 14b shows the measured normalized acceleration CSD between sensor \#1 and all the other sensors. Strong spatial coherences of the vibratory field at many frequencies can be observed. In general, the frequencies related to these strong coherences correspond to the pseudo-axial modes. One can notice some similarities between the patterns shown in Fig. 14a and Fig. 14b. Above $1.5 \mathrm{kHz}$, the measured coherence between sensors is less important than that estimated by the model as shown in Fig. 13. This could be attributed to the differences in the TBL excitation in the numerical simulation and experiment. A flow rate of $140 \mathrm{l} / \mathrm{s}$ was set in the experiment to ensure that the flow was turbulent in the pipe. Moreover, in the upstream of the test section, a $4 \mathrm{~m}$ long pipe and a perforated plate flow conditioner were used to stabilize the turbulent flow. A $1.5 \mathrm{~m}$ long pipe was also used for the discharge before 
the acoustic balloon. However, the wall pressure spectrum induced by the turbulent flow was not measured. Therefore, it cannot confidently be stated that the Chase TBL model considered in the numerical modelling is well adapted for describing the wall pressure field in the full frequency range.

To summarize and in spite of reasonable differences, it seems apparent that the proposed model is able to represent the main trends of the vibratory behaviour of a fluid filled pipe coupled with two flanges. In particular, the model can justify the strong spatial coherences of the vibratory field observed in the experimental measurements, which have been proved to be associated to frequencies related to the pseudo-axial modes. Further experimental validation against experimental data from the Pennsylvania State University [33] is provided in Appendix B, showing also good matching. Moreover, a previous satisfactory validation was reported in [43], for the case of radial mechanical point force excitation with an impedance hammer, in the laboratory mock-up of Fig. 1.

\subsection{Influence of the system parameters on the spatial coherence of the vibratory field}

\subsubsection{Influence of the shell thickness}

Next, the influence of some system parameters on the spatial coherence of the vibratory field are investigated starting with the shell thickness. Only the shell without the stiffeners is considered in this section. Note that the shell thickness $h$ appears in the elements of the spectral

Flügge operator $\tilde{\tilde{\mathcal{L}}}\left(k_{x}, n\right)$ presented in Appendix A. As introduced in Eq. (13), the factor $\gamma$ also involves $h$ but it could be easily incorporated in the Flügge matrix as a $\gamma^{-1}$ factor. The elements in $\tilde{\tilde{\mathcal{L}}}\left(k_{x}, n\right)$ are involved in Eq. (19) which provide the shell spectral radial displacement $\tilde{W}_{F_{(x, \theta, \omega)}}$ when submitted to the force in Eq. (25) for $\tilde{F}_{1}=\tilde{F}_{2}=0$. Given that the sensitivity functions are computed as $\tilde{\tilde{H}}_{w}=R \tilde{\tilde{W}}_{F_{(x, \theta, \omega)}}$, it is clear that they will become affected by the shell thickness and so does the vibration field of the shell induced by the TBL which is finally computed with Eq. (3), as that equation contains $\tilde{\tilde{H}}_{w}$.

In Fig. 15 we present the normalized CSD of the acceleration between sensor \#1 and all other sensors for the cylindrical shell without the ring stiffeners and for two different shell thicknesses of $4 \mathrm{~mm}$ and $16 \mathrm{~mm}$. These thicknesses respectively correspond to half and twice the thickness of the shell studied in the previous sections (i.e. $8 \mathrm{~mm}$ ). For the $4 \mathrm{~mm}$-thick shell, the cut-on frequencies in the frequency band of [100 Hz- $2 \mathrm{kHz}]$ can be found at $150 \mathrm{~Hz}, 453$ 
$\mathrm{Hz}, 918 \mathrm{~Hz}$ and $1546 \mathrm{~Hz}$, corresponding to the circumferential order $n$ ranging from 2 to 5 . For the $16 \mathrm{~mm}$-thick shell, there is only one cut-on frequency in the frequency band of interest, namely $784 \mathrm{~Hz}$ for $n=2$. Fig. 15 shows that these cut-on frequencies play a significant role in the formation of the patterns observed in the spatial coherence. As already stated in the previous section, the coherence between sensors is close to one for these frequencies but decreases between some sensors for the remaining frequencies. The distances between weak-coherence sensors (i.e. black areas on the figure) are related to the wavelength of the propagative waves for the corresponding circumferential order at the considered frequency.

\subsubsection{Influence of the stiffness of the flanges}

In what follows, let us consider the shell coupled to the stiffeners and study the influence of the stiffness of the ring flanges on the spatial coherence of the vibratory field. From a modelling point of view, the stiffness of the flanges for a given material is controlled by the moment of inertia of the ring cross-section of width $l_{r}$ and height $h_{r}$. The latter appear in the expression for the circumferential admittance for the ring stiffener $\tilde{Y}_{r}(n)$ in Eq. (24). A change in the ring cross-section will thus affect $\tilde{Y}_{r}(n)$, which in turn will influence the contact forces in Eq. (11). That will automatically impact the computation of the shell circumferential sensitivity functions according to the reasoning in section 2.2.4 and finally the vibration field of the shell when excited by the TBL through Eq. (3).

To test the impact of the flange stiffness two new configurations are considered:

- First configuration: the fluid filled cylindrical shell is coupled to two totally rigid stiffeners having null circumferential admittance (i.e. $\left.\tilde{Y}_{r}(n)=0\right)$.

- Second configuration: the fluid filled cylindrical shell is coupled to two ring stiffeners having a rectangular cross-section of $10 \times 30 \mathrm{~mm}^{2}$ (instead of the previous one of $40 \times$ $\left.60 \mathrm{~mm}^{2}\right)$.

Except for the above changes, all other parameters in the two new configurations (e.g., characteristics of the cylindrical shell and fluid, distance between the stiffeners, etc.) remain the same as for the previous simulations.

Beginning with the second configuration, Fig. 16 compares the amplitudes of the stiffeners' admittances with those of the fluid filled shell. These amplitudes are higher than those of the nominal configuration shown in Fig. 5. This is because the new stiffeners are softer than those of the nominal configuration in the entire frequency range. On the other hand, one can observe that for $n=2$ the shell admittance is greater than the stiffener admittance for 
frequencies below $460 \mathrm{~Hz}$ and that the opposite occurs for frequencies above $460 \mathrm{~Hz}$. This indicates that for the circumferential order $n=2$, the shell is more rigid than the stiffeners above $460 \mathrm{~Hz}$, while for the nominal configuration, the shell was softer than the stiffeners regardless of frequency. For $n=3$, the shell is softer than the stiffeners except for frequencies above 1864 $\mathrm{Hz}$.

Fig. 17 depicts the mean ASD of the accelerations measured by the virtual line array of 25 sensors for the two new configurations. For the shell with totally rigid stiffeners, one can realize that additional peaks appear compared to the nominal case shown in Fig. 7b. For instance, the two extra peaks at $208 \mathrm{~Hz}$ and $892 \mathrm{~Hz}$ can be attributed to $n=1$. These peaks do not exist for the nominal configuration because the ring stiffeners were softer than the shell. This was highlighted in Fig. 5. On the contrary, for the second configuration, the peaks between $460 \mathrm{~Hz}$ and $1000 \mathrm{~Hz}$ have disappeared compared to the initial configuration. As it has been previously mentioned, for the second configuration and $n=2$ the shell is more rigid than the stiffeners beyond $460 \mathrm{~Hz}$, which explains the vanishing of the peaks. Above $1072 \mathrm{~Hz}$, the vibratory behaviour is mainly controlled by the circumferential order $n=3$. As the shell is softer than the stiffener for this order for frequencies below $1864 \mathrm{~Hz}$, the peaks induced by the pseudo axial modes manifest with amplitudes which are, however, lower than those of the initial configuration (in particular for the higher part of the frequency range).

The spatial coherence of the vibratory field can be examined by plotting the normalized CSD of acceleration, as shown in Fig. 18. For the first configuration, one notices strong coherences for the frequencies corresponding to the supplementary peaks observed in Fig. 17, in particular at $208 \mathrm{~Hz}$ and $892 \mathrm{~Hz}$. For the second configuration, the results for frequencies between $460 \mathrm{~Hz}$ and $1072 \mathrm{~Hz}$ are similar to those of the shell without stiffeners as shown in Fig. 13a, whereas above $1072 \mathrm{~Hz}$ they are similar to those for the nominal case, as shown in Fig. 13b.

\subsection{Auto and normalized cross-spectrum density for the virtual ring wire sensor}

Before concluding this study, let us next change the type of virtual sensors' array and consider a piezoelectric wire wound around the pipe an integral number of times (see [41] and also the works in $[44,45])$. This type of sensor is only sensitive to axisymmetric waves [46], which means it can only detect the component of the radial vibratory field corresponding to the circumferential order $n=0$. Here we consider the case of 25 wire rings whose axial positions 
coincide with those of the accelerometers in the line array of the previous section, i.e., $x_{m}=0.06+0.04 m, \forall m \in\{1, \ldots, 25\}$.

The normalized CSD of the radial acceleration between the $i$-th and $j$-th wires of the new virtual array can be obtained from,

$$
\Gamma_{i j}^{\gamma(n=0)}(\omega)=\frac{\left|S_{\gamma \gamma}^{n=0}\left(x_{i}, x_{j}, \omega\right)\right|}{\sqrt{S_{\gamma}^{n=0}\left(x_{i}, x_{i}, \omega\right) S_{\gamma \gamma}^{n=0}\left(x_{j}, x_{j}, \omega\right)}},
$$

where $S_{\gamma /}^{n=0}$ is the CSD of the radial acceleration considering only the contribution of the circumferential order $n=0$ in Eq. (7) and we remember again that $S_{\gamma}=\omega^{4} S_{w w}$.

In Fig. 19, we compare the ASD mean value of the radial acceleration "measured" by the virtual wire sensor array, once more for the shell with and without stiffeners. The ASD levels are much lower than those registered by the classical accelerometer line array in Fig. 7. This is consistent with the analysis of the operational dispersion curves of Section 3.2, which are reflected in Fig. 6. On the other hand, Fig. 19 also reveals that stiffeners have negligible effect on the order $n=0$ contribution to the vibratory field. This may be surprising since in Figs. $5 b-c$ the stiffener admittances for $n=0$ are one order of magnitude lower than the shell admittances (i.e. nearly $2.6 \times 10^{-11} \mathrm{~m} / \mathrm{N}$ for the stiffeners against $1.6 \times 10^{-10} \mathrm{~m} / \mathrm{N}$ for the shell). One would therefore expect that the flanges could block the vibrations of the shell to some extent. As discussed in Section 3.1, however, for this circumferential order the behaviour of the fluid filled shell is mainly controlled by the fluid rather than by the shell (see also [40, 44]). Consequently, neither the latter nor the stiffeners have any influence on the filled fluid shell dynamics for $n=0$, as observed in Fig. 19.

The normalized CSD function of the radial acceleration measured by the virtual wire sensor array is depicted in Fig. 20. Only small differences are observed between the shell with and without flanges. The signals measured by the ring wire array are clearly less coherent than those obtained with the classical accelerometer line array. This difference can be explained by the fact that the strong coherences observed with the line array were mostly related to the vibroacoustic characteristics of the shell (cut-on frequency of the propagative wave, pseudo axial modes, etc.), whereas as said before and illustrated in Fig. 6a, for $n=0$ the vibroacoustics of the system is dominated by the fluid. Below $600 \mathrm{~Hz}$, the coherences in Fig. 20 between the ring wire sensors are too small to make them suitable for classical beamforming strategies for noise source detection. Above $600 \mathrm{~Hz}$, the coherences become more significant. The coherence plot presents a pattern of curves, which for a fixed frequency, exhibit maxima and minima. The distance between a pair of maxima (or minima) corresponds to half wavelength of the dominant 
propagative wave (roughly half of the acoustic wavelength in water). For instance, the wavelength is $0.75 \mathrm{~m}$ at $2 \mathrm{kHz}$, and the distance between maxima at that frequency in Fig. 20 is $0.36 \mathrm{~m}$.

\section{Conclusions}

In this paper we have proposed a semi-analytical model for a fluid filled cylindrical shell coupled to two ring stiffeners and excited by an internal, homogeneous, turbulent boundary layer (TBL). The model has served to determine the spatial coherence of the shell vibratory field along a line of virtual point accelerometers. It has been shown that, quite unexpectedly, a weakly spatially correlated TBL in the interior of a pipe can give place to a strongly spatially correlated vibratory field on the pipe surface. The spatial coherence obviously varies from one frequency to another and depends on the vibroacoustic behaviour of the fluid-filled shell. The frequencies with stronger coherence are those corresponding to the cut-on frequencies of the circumferential orders, as well as to the resonant frequencies of the pseudo axial modes (in the case of stiffened shells).

The herein developed model is based on the computation of circumferential admittances of the fluid-filled shell using a spectral approach. Only two ring flanges have been considered in the formulation, though extension to further configurations with more stiffeners is quite straightforward. The circumferential admittance approach (CAA) has allowed us to analyse the mechanical characteristics of the shell and the stiffeners. Their coupling has been assumed to only take place in the radial direction, which suffices for the purpose of this study, as shown by the validation with experimental results reported in subsection 3.5 and Appendix B. Also, the influence of some system parameters like the shell thickness and the stiffness of the flanges on the spatial coherence has been investigated.

The current work may have significant applications for vibration beamforming techniques. In fact, it has provided an explanation to the unforeseen experimental results in [22], where a beamforming linear array was used to explore ways of detecting sodium-water reactions in the steam generator unit of a liquid sodium fast reactor. Moreover, it has also been shown that if one alternatively resorts to ring wire piezoelectric sensors, which can only detect the component $n=0$ of the vibratory field, the coherence between sensors become much weaker than when using standard accelerometer line arrays. This is explained by the fact that, for the zero-th order, the pipe vibratory field is driven by the axial propagation of waves within the fluid rather than by the shell and stiffener properties. Given that classical beamforming requires 
uncorrelated background noise, the use of an array of these piezoelectric wires instead of classical accelerometers may lead to better performance for noise source detection. However, further investigations should be carried out to verify that ring wires could be as good as accelerometer line arrays to locate acoustic sources in the case of no background noise. Conversely, as the signals measured by a line array of accelerometers exhibit strong coherence in the presence of the TBL background noise, the use of advanced beamforming treatments based on it's a priori knowledge, like the MaxSNR [22], could be a good option.

\section{Appendix A: TBL and shell models}

The cross spectral density of the Chase TBL model is given by (see e.g., [13]),

$$
S_{p p}^{t b l}\left(k_{x}, k_{y}, \omega\right)=\frac{\rho_{0}^{2} U_{\tau}{ }^{3}}{\left[K_{+}^{2}+\left(b \delta_{t b l}\right)^{-2}\right]^{5 / 2}}\left\{C_{M} k_{x}^{2}+C_{T} K^{2}\left[\frac{K_{+}^{2}+\left(b \delta_{t b l}\right)^{-2}}{K^{2}+\left(b \delta_{t b l}\right)^{-2}}\right]\right\}
$$

with $K_{+}^{2}=\left(\omega-U_{c} k_{x}\right)^{2} /\left(h U_{\tau}\right)^{2}+K^{2}, K^{2}=k_{x}{ }^{2}+k_{y}{ }^{2}$ and recommended parameters $b \approx 0.75$, $C_{M} \approx 0.1553, C_{T} \approx 0.0047$ and $h=3$ (see e.g., [13]).

On the other hand, the spectral Flügge matrix $\tilde{\tilde{\mathcal{L}}}\left(k_{x}, n\right)$ implemented in this work reads,

$$
\tilde{\tilde{\mathcal{L}}}\left(k_{x}, n\right)=\left[\begin{array}{lll}
\tilde{\tilde{Z}}_{U U} & \tilde{\tilde{Z}}_{U V} & \tilde{\tilde{Z}}_{U W} \\
\tilde{\tilde{Z}}_{U V}^{*} & \tilde{\tilde{Z}}_{V V} & \tilde{\tilde{Z}}_{V W} \\
\tilde{\tilde{Z}}_{U W}^{*} & \tilde{\tilde{Z}}_{V W}^{*} & \tilde{\tilde{Z}}_{W W}
\end{array}\right],
$$

with

$$
\begin{aligned}
& \tilde{\tilde{Z}}_{U U}=-R^{2}\left(k_{l}^{2}-k_{x}^{2}\right)+n^{2} \frac{1-v}{2}\left(1+\beta^{2}\right), \quad \tilde{\tilde{Z}}_{U V}=n R \frac{1+v}{2} k_{x}, \\
& \tilde{\tilde{Z}}_{U W}=\mathrm{i} k_{x}\left[-R v+\beta^{2}\left(-R^{3} k_{x}^{2}+n^{2} R \frac{1-v}{2}\right)\right], \quad \tilde{\tilde{Z}}_{V V}=R^{2} \frac{1-v}{2} k_{x}^{2}\left(1+3 \beta^{2}\right)+n^{2}-R^{2} k_{l}^{2}, \\
& \tilde{\tilde{Z}}_{V W}=-\mathrm{i} n\left(1+\beta^{2} R^{2} \frac{3-v}{2} k_{x}^{2}\right), \quad \tilde{\tilde{Z}}_{W W}=1+\beta^{2}\left(R^{4} k_{x}^{4}+2 n^{2}\left(R^{2} k_{x}^{2}-1\right)+n^{4}+1\right)-R^{2} k_{l}^{2},
\end{aligned}
$$

where $\beta=\frac{h}{R \sqrt{12}}$ (remember $h$ is the shell thickness) and $k_{l}=\omega \sqrt{\frac{\rho\left(1-v^{2}\right)}{E}}$ is the wavenumber for compressional waves and the asterisk denotes the complex conjugate.

\section{Appendix B: Numerical - experimental comparison}

In this appendix, we compare the results of the model developed in Section 2 with the experimental results in Fig. 7 of reference [33]. The experiment in that work was conducted in the Garfield Thomas Water Tunnel, at the Applied Research Lab in Penn State University. The 
test pipe section comprised a cylindrical shell of $0.0032 \mathrm{~m}$ thickness, a length of $0.61 \mathrm{~m}$ and a radius of $0.156 \mathrm{~m}$. The pipe was made of aluminum and two large blocks (also made of aluminum) were used at both ends of the test section for attaching the pipe to the floor. To simulate simply supported conditions, circumferential V-shaped grooves were machined around the pipe, at the ends of the test-section. A ring of 12 accelerometers was used to measure the pipe vibrations. Three runs were measured with the same flow conditions, moving a ring array to 3 different axial locations $(x / L=1 / 2,1 / 3,1 / 6$, where $L$ is the length of the test section and $x=0$ corresponds to its upstream end). More details on this experiment can be found in [33]. The measured acceleration spectra in response to the turbulent flow for the three different positions of the ring array were averaged. The results for a flow speed of $6.1 \mathrm{~m} / \mathrm{s}$ were reported in Fig. 7 of [33] and have been included in Fig. B.1.a. The dashed lines in the figure correspond to directly measured values, while the solid ones stand for values once applied a noise removing technique. Moreover, the contributions of the first 5 circumferential modes were extracted from the measured data and plotted in the figure. The modal orders associated to each mode have been indicated; the first number designates the circumferential order and the second one the axial order.

For validation, we have reproduced the above experimental set-up with the proposed semi-analytical model. The ring stiffeners have been positioned at $x=0 \mathrm{~m}$ and $x=0.61 \mathrm{~m}$ to represent the effects of the aluminum blocks. The geometry of the blocks does not exactly match the one considered for the ring stiffeners in this paper. However, considering their characteristics (see [33]), one can suppose that they are much stiffer in the radial direction than the cylindrical shell. Ring stiffeners of rectangular cross section $(0.05 \mathrm{~m} \times 0.01 \mathrm{~m})$ allow us mimicking this property. For the simulations, we have considered the following material data for aluminum, $\rho=2700 \mathrm{~kg} / \mathrm{m}^{3}, E=59 \mathrm{GPa}, v=0.33, \eta=0.001$, whereas for water we have taken $\rho_{0}=1000 \mathrm{~kg} / \mathrm{m}^{3}, c_{0}=1500 \mathrm{~m} / \mathrm{s}$ and $\eta_{0}=0.001$. Concerning the TBL excitation, the wall pressure field is represented by the Chase model [13] (see Appendix A). The flow speed, $U_{0}$, has been set to $6.1 \mathrm{~m} / \mathrm{s}$, the TBL thickness has been chosen equal to the shell radius and the convective and friction velocities are respectively given by $U_{c} \approx 0.7 U_{0}$ and $U_{\tau} \approx 0.032 U_{0}$.

The results of the simulations are plotted in Fig. B.1.b. The comparison with the experimental results in Fig. B.1.a is quite satisfactory. There is a good agreement between the measured and predicted vibratory levels. In addition, the positions of the frequency peaks are well predicted by the model. The dominant contributions of each circumferential mode are also well captured in the simulation and they are consistent with the experimental data. Some 
discrepancies in vibratory levels are appreciated, which can be attributed to uncertainties in the description of the wall pressure field by the Chase model (as highlighted in [33]). The most important difference appears for the peak level close to $280 \mathrm{~Hz}$, associated to mode $(2,1)$. One possible explanation is that this mode occurs at low frequencies and may had been more affected by the external conditions of the test rig than the higher order modes. For instance, in the experiment, the external portions of the pipe are significantly thicker than the region of the test section $(0.0064 \mathrm{~m}$ against $0.0032 \mathrm{~m})$, whereas in the simulation a $0.0032 \mathrm{~m}$ cylindrical shell was considered. Overall, however, we believe the comparison between simulated and measured data is very satisfactory and validates the modelling approach proposed in this paper.

\section{Acknowledgments}

This work was performed within the framework of the LABEX CeLyA (ANR-10-LABX-0060) of Université de Lyon, within the program « Investissements d'Avenir » (ANR-16-IDEX-0005) operated by the French National Research Agency (ANR).

\section{References}

[1] Flinovia - Flow induced noise and vibrations issues and aspects: A focus on measurement, modeling, simulation and reproduction of the flow excitation and flow induced response (Springer International Publishing Switzerland, 2015, pp. 357).

[2] Flinovia - Flow induced noise and vibrations issues and aspects II: A focus on measurement, modeling, simulation and reproduction of the flow excitation and flow induced response (Springer International Publishing Switzerland, 2019, pp. 344).

[3] W. A. Strawderman, Turbulence-induced plate vibrations: An evaluation of finite- and infinite-plate models, J. Acoust. Soc. Am. 46 (1969) 1294-1295.

[4] W. A. Strawderman and R. A. Christman, Turbulence-induced plate vibrations: Some effects of fluid loading on finite and infinite plates, J. Acoust. Soc. Am. 52 (1971) $1537-1552$.

[5] C. Maury, P. Gardonio, S.J. Elliott, A wavenumber approach to modelling the response of a randomly excited panel. Part I: general theory, J. Sound Vib. 252 (2002) 83-113.

[6] S. De Rosa, F. Franco, Exact and numerical responses of a plate under a turbulent boundary layer excitation, J. Fluids Struc. 24 (2008) 212-230. 
[7] F. Franco, S. De Rosa, E. Ciappi, Numerical approximations on the predictive responses of plates under stochastic and convective loads, J. Fluids Struc. 42 (2013) 296-312

[8] M. Karimi, P. Croaker, L. Maxit, O. Robin, A. Skvortsov, S. Marburg, N. Kessissoglou, a hybrid numerical approach to predict the vibrational responses of panels excited by a turbulent boundary layer, J. Fluids. Struct. 92 (2020) 102814.

[9] E. Ciappi, S. De Rosa, F. Franco, P. Vitiello, M. Miozzi, On the dynamic behavior of composite panels under turbulent boundary layer excitations, J. Sound Vib. 364 (2016) 77-109.

[10] C. Marchetto, L. Maxit, O. Robin, A. Berry, Experimental prediction of the vibration response of panels under a turbulent boundary layer excitation from sensitivity functions, J. Acoust. Soc. Am. 143 (2018) 2954-2964.

[11] G. M. Corcos, Resolution of pressure in Turbulence, J. Acoust. Soc. Am. 35 (1963) 192-199.

[12] R. H. Mellen, Wave-vector filter analysis of turbulent flow, J. Acoust.Soc. Am. 95 (1994) 1671-1673.

[13] D.M. Chase, The character of the turbulent wall pressure spectrum at subconvective wavenumbers and a suggested comprehensive model, J. Sound Vib. 112 (1987) 125147.

[14] W. R. Graham, A comparison of models for the wavenumber-frequency spectrum of turbulent boundary layer pressures, J. Sound Vib. 206 (1997) 541-565.

[15] M.P., Norton, M.K. Bull, Mechanisms of the generation of external acoustic radiation from pipes due to internal flow disturbances, J. Sound Vib. 94 (1984) 105-146.

[16] C. Durant, G. Robert, P.J.T. Filippi, P.O. Mattei, Vibroacoustic response of a thin cylindrical shell excited by a turbulent internal flow: comparison between numerical prediction and experimentation, J. Sound Vib. 229 (2000) 1115-1155.

[17] Y. Li, Y. Zhang, D. Kennedy, Random vibration analysis of axially compressed cylindrical shells under turbulent boundary layer in a symplectic system. J. Sound Vib. 406 (2017) 161-180.

[18] L. Maxit, M. Karimi, V. Meyer, N. Kessissoglou, Vibroacoustic responses of a heavy fluid loaded cylindrical shell excited by a turbulent boundary layer, J. Fluids. Struct. $92(2020) 102758$. 
[19] L. Maxit, V. Denis, Prediction of flow induced sound and vibration of periodically stiffened plate, J. Acoust. Soc. Am. 133 (2013) 146-160.

[20] C. Marchetto, L. Maxit, O. Robin, A. Berry, Vibroacoustic response of panels under diffuse acoustic field excitation from sensitivity functions and reciprocity principles, J. Acoust. Soc. Am. 141 (2017) 4508-4521.

[21] L. Maxit, O. Guasch, V. Meyer, M. Karimi, Noise radiated from a periodically stiffened cylindrical shell excited by a turbulent boundary layer, J. Sound Vib. 466 (2020) 115016.

[22] S. Kassab, F. Michel, L. Maxit, Water experiment for assessing the vibroacoustic beamforming gain in the framework of the acoustic leak detection for the sodiumheated steam generator, Mech. Syst. Signal Process 134 (2019) 106332.

[23] M.J. Brennan, M. Karimi, J.M. Muggleton, F.C.L. Almeida, F. Kroll de Lima, P.C. Ayala, D. Obata, A.T. Paschoalini and N. Kessissoglou, On the effects of soil properties on leak noise propagation in plastic water distribution pipes, J. Sound Vib. 427 (2018) 120-133.

[24] M. Cavaro, C. Payan, J.P. Jeannot, Towards the bubble presence characterization within the SFR liquid sodium, In: Proceedings of ANIMMA International Conference, Marseille, France, vol. 1127, June, 2013.

[25] Y. Chikazawa, Acoustic leak detection system for sodium-cooled reactorsteam generators using delay-and-sum beamformer, J. Nucl. Sci. Technol. 47 (2010), 103110.

[26] T. Kim, V.S. Yugay, J. Jeong, J. Kim, B. Kim, T. Lee, Y. Lee, Y. Kim, D. Hahn D., Acoustic Leak Detection Technology for Water/Steam Small Leaks and Microleaks Into Sodium to Protect an SFR Steam Generator. Nucl. Technol. 170 (2010) 360-369.

[27] Y. Chikazawa, T. Yoshiuji, Water experiment on phased array acoustic leak detection system for sodium-heated steam generator, Nucl. Eng. Des. 289 (2015) 1-7.

[28] J. Moriot, L. Maxit, J.L. Guyader, O. Gastaldi, J. Périsse, Use of beamforming for detecting an acoustic source inside a cylindrical shell filled with a heavy fluid. Mech. Syst. Signal Process 52-53 (2015) 645-662.

[29] B. Van Veen, K. Buckley, Beamforming: a versatile approach to spatial filtering. IEEE ASSP magazine 5 (1988), 4-24.

[30] L. Maxit, J.M. Ginoux, Prediction of the vibro-acoustic behavior of a submerged shell non periodically stiffened by internal frames, J. Acoust. Soc. Am. 128 (2010) 137-151. 
[31] L. Maxit, Scattering model of a cylindrical shell with internal axisymmetric frames by using the circumferential admittance approach, Appl. Acoust. 80 (2014) 10-22.

[32] V. Meyer, L. Maxit, J.-L. Guyader, T. Leissing, Prediction of the vibroacoustic behaviour of a submerged shell with non-axisymmetric internal substructures by a condensed transfer function method, J. Sound Vib. 360 (2016) 260-276.

[33] W. Bonness, D. Capone, S. Hambric, Low-wavenumber turbulent boundary layer wallpressure measurements from vibration data on a cylinder in pipe flow, J. Sound Vib. 329 (2010) 4166-4180.

[34] L. Maxit, Simulation of the pressure field beneath a turbulent boundary layer using realizations of uncorrelated wall plane waves, J. Acoust. Soc. Am. 140 (2016) 12681285.

[35] S. Hambric, Y. Hwang, W. Bonness, Vibrations of plates with clamped and free edges excited by low-speed turbulent boundary layer flow, J. Fluids Struct. 19 (2004) 93110.

[36] Leissa, A.W., 1973. Vibration of shells. Washington: Scientific and Technical Information Office, National Aeronautics and Space Administration.

[37] M.C. Junger, D. Feit, Sound, structures, and their interaction, Second edition, MIT Press, 1986.

[38] C.R. Fuller, F.J. Fahy, Characteristics of wave propagation and energy distributions in cylindrical elastic shells filled with fluid. J. Sound Vib. 81 (1982) 501-518.

[39] C.R. Fuller, The input mobility of an infinite circular cylindrical elastic shell filled with fluid. J. Sound Vib. 87 (1983) 409-427.

[40] M. Tran-Van-Nhieu, Scattering from a ribbed finite cylindrical shell. The Journal of the Acoustical Society of America, 110 (2001), 2858-2866.

[41] J.M. Muggleton, M.J. Brennan, the design and instrumentation of an experimental rig to investigate acoustic methods for the detection and location of underground piping systems, Appl. Acoust. 69 (2008) 1101-1107.

[42] M. Karimi, L. Maxit, P. Croaker, O. Robin, A. Skvortsov, S. Marburg, N, Atalla, N. Kessissoglou, Analytical and numerical prediction of acoustic radiation from a panel under turbulent boundary layer excitation, J. Sound Vib, 479 (2020) 115372.

[43] S. Kassab, Vibroacoustic beamforming for the detection of an acoustic monopole inside a thin cylindrical shell coupled to a heavy fluid: Numerical and experimental 
developments, PhD thesis, Université de Lyon, INSA Lyon, Villeurbanne, France, 2018, 182 p. (in French).

[44] J.M. Muggleton, M.J. Brennan, R.J. Pinnington, Y. Gao, a novel sensor for measuring the acoustic pressure in buried plastic water pipes, J. Sound Vib. 295 (2006) 10851098.

[45] G. Pavic, F. Chevillotte, Cavitation monitoring in piping systems, Proceedings of ISMA 2012, Leven, Belgium, September 2012.

[46] R.J. Pinnington, A.R. Briscoe, Externally applied sensor for axisymmetric waves in a fluid filled pipe, J. Sound Vib. 173 (1994) 503-516. 


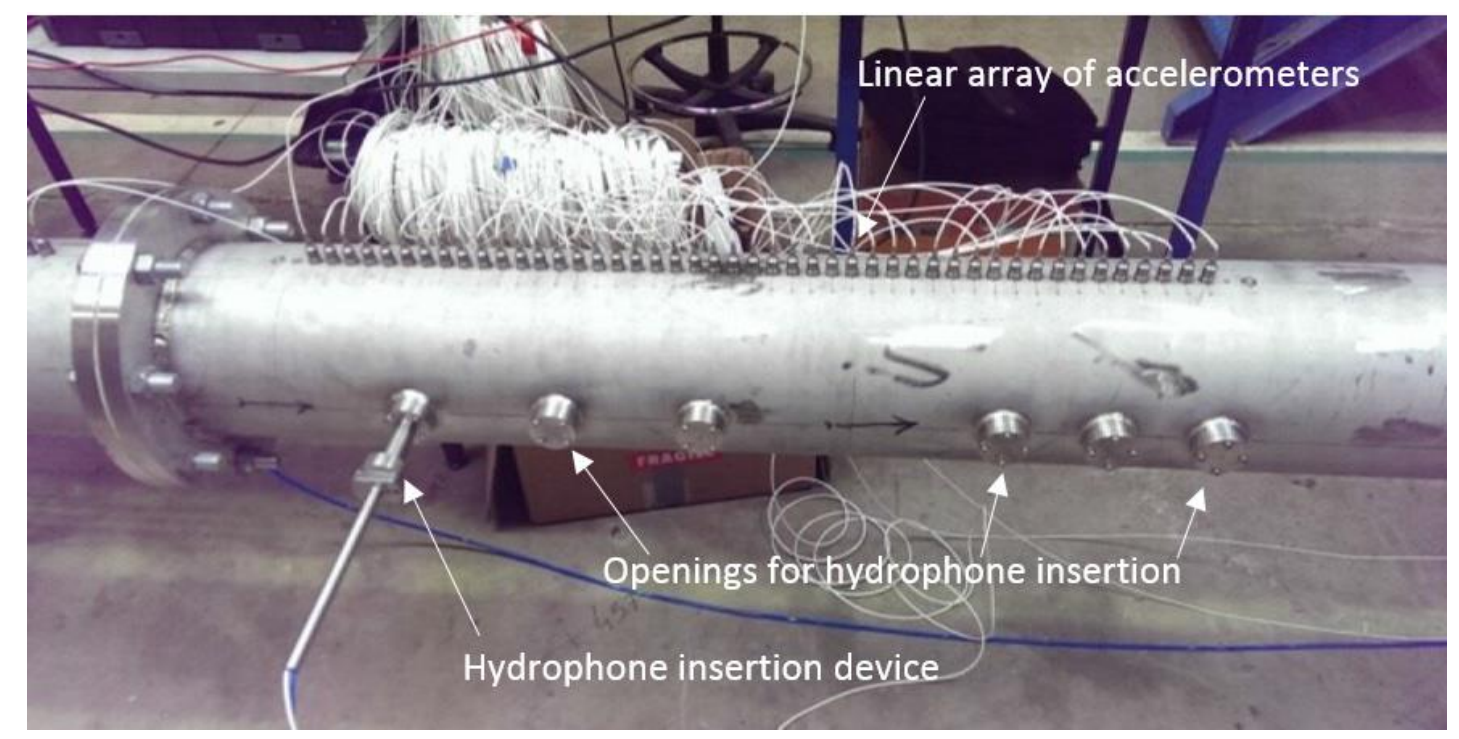

Figure 1. Experimental set-up for assessing the performance of the vibroacoustic beamforming [22]. 


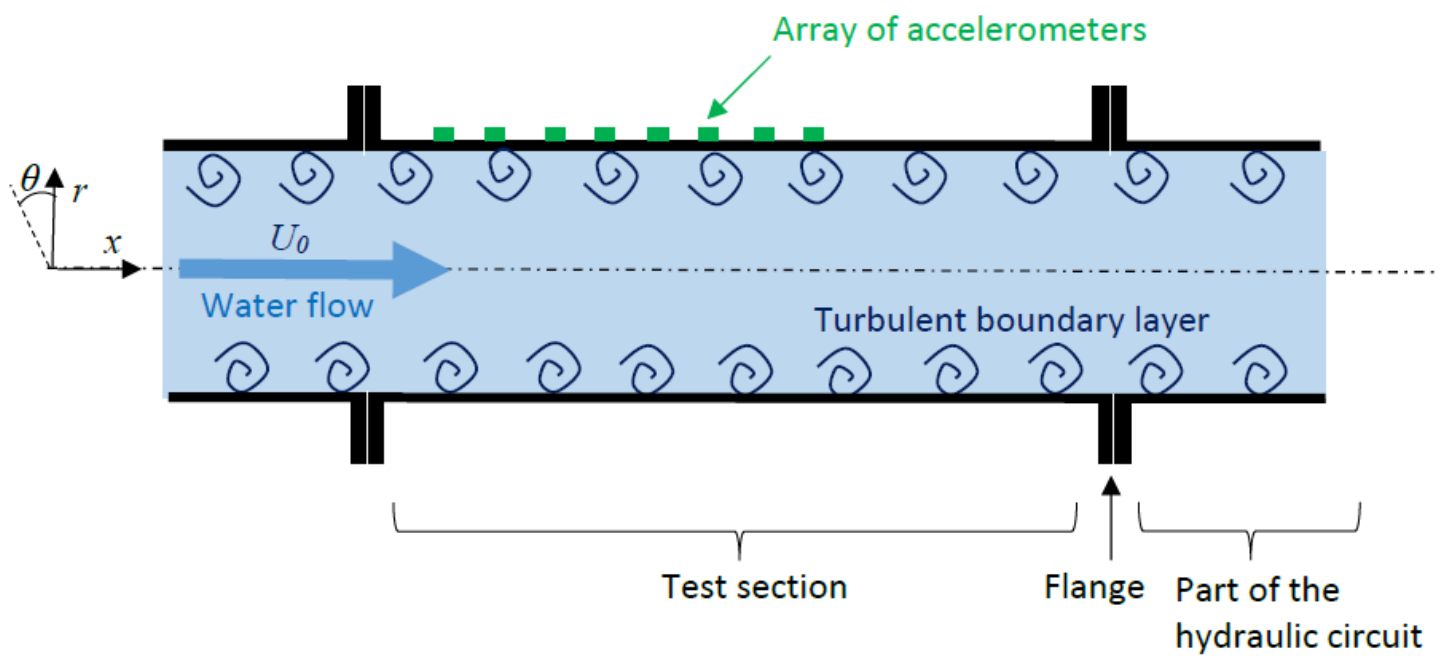

Figure 2. Schematic representation of the second pipe configuration: An infinite cylindrical shell coupled to two ring stiffeners and filled with a water turbulent flow. 
First CAA calculation

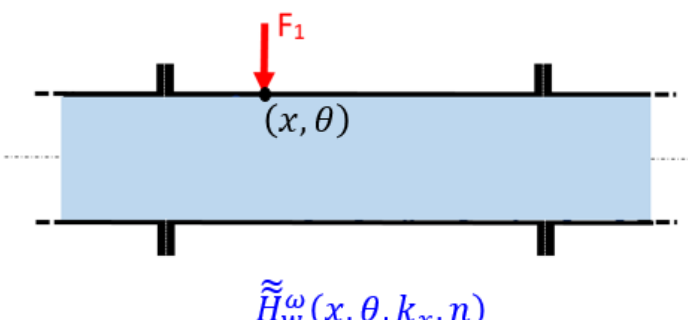

Second CAA calculation

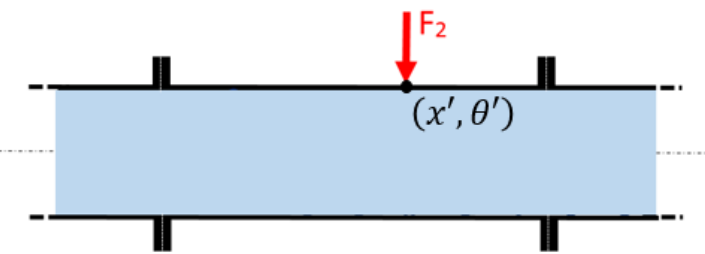

$\widetilde{H}_{w}^{\omega}\left(x^{\prime}, \theta^{\prime}, k_{x}, n\right)$

WPF model $\rightarrow \phi_{p p}^{t b l} \longrightarrow$ Eq. (7)

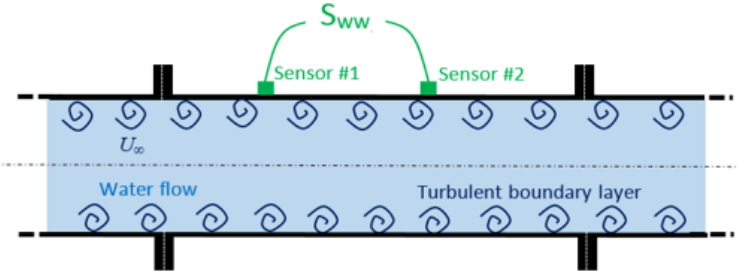

Figure 3. Schematic diagram describing how to evaluate the CSD of the accelerations between two sensors. 


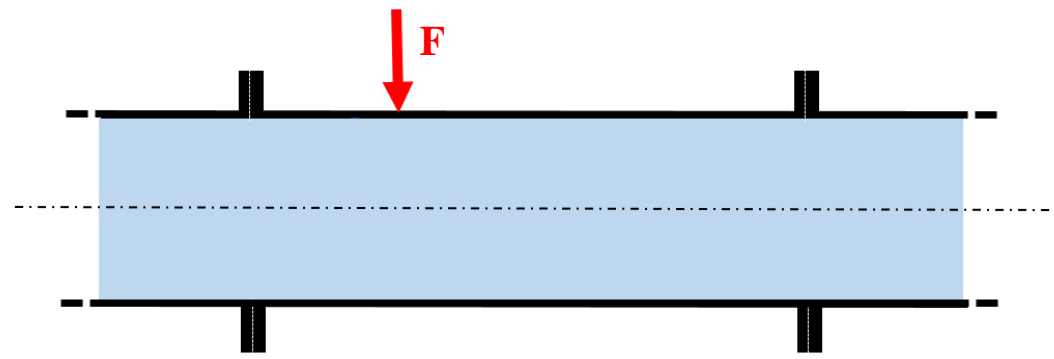

(a)
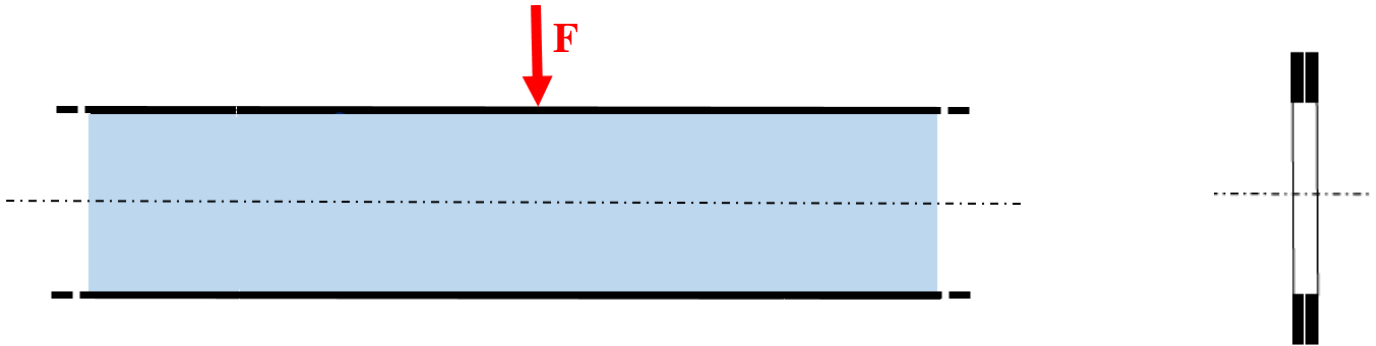

(b)

Figure 4. (a) Global problem: fluid filled cylindrical shell coupled two ring stiffeners.

(b) Partitioning of the vibroacoustic problem into subsystem \#1 (fluid filled cylindrical shell) and subsystem \#2 (ring stiffeners). Red arrows represent radial point forces. 


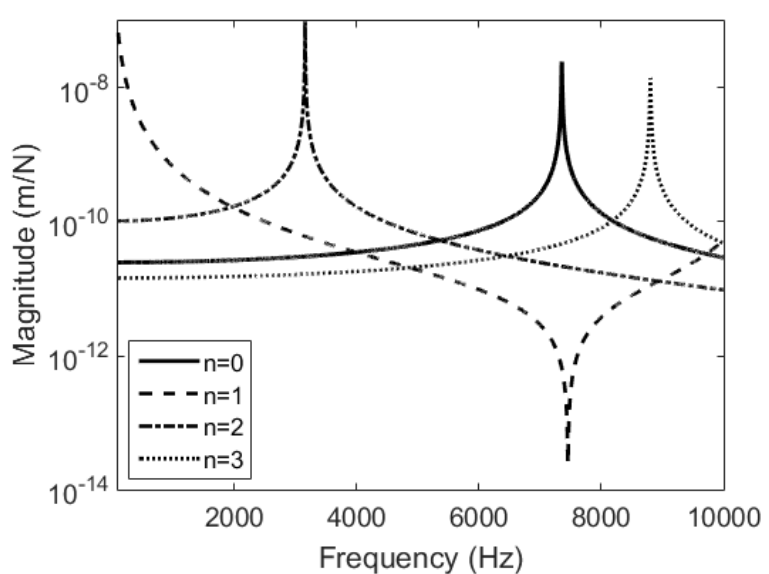

(a)

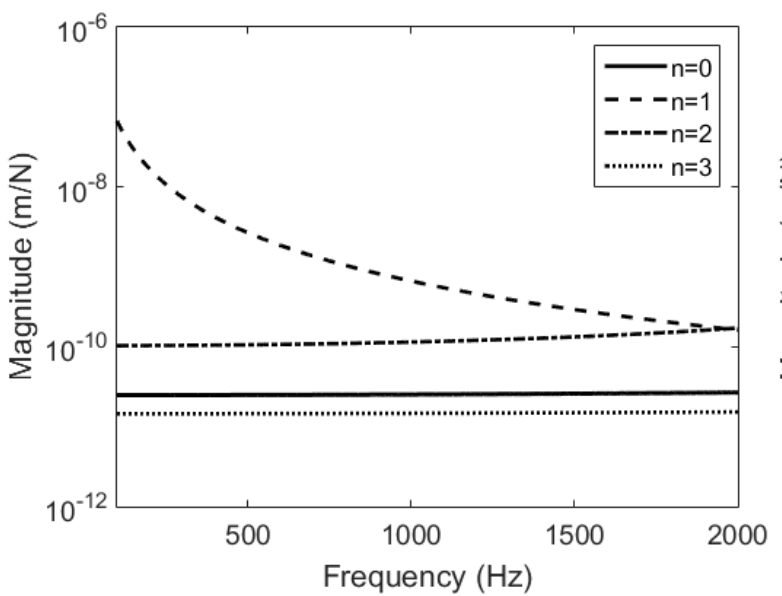

(b)

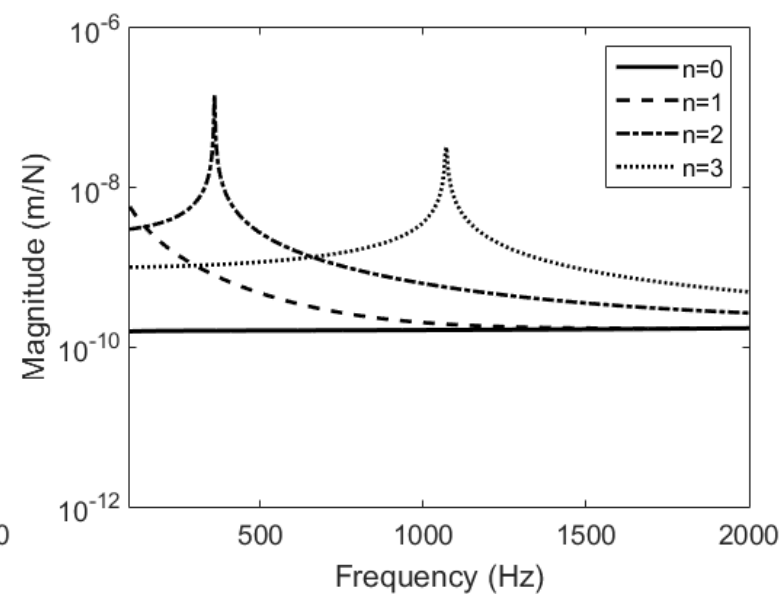

(c)

Figure 5. Amplitude of the circumferential admittances versus frequency for different circumferential orders in the cases of, (a) ring stiffener in the large frequency range $[110,000]$ $\mathrm{Hz}$, (b) zoom of subfigure (a) in the frequency range of interest [100 2000] Hz, c) fluid filled cylindrical shell in the frequency range of interest [100 2000] Hz. 


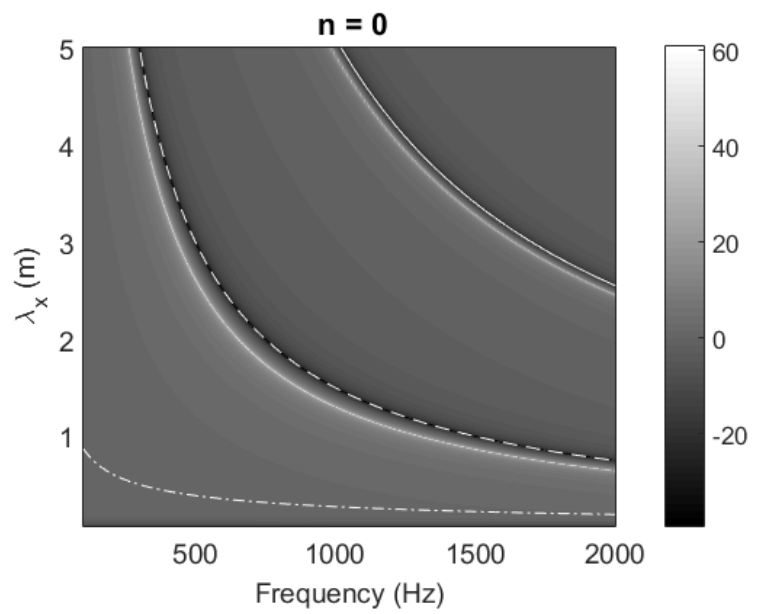

(a)

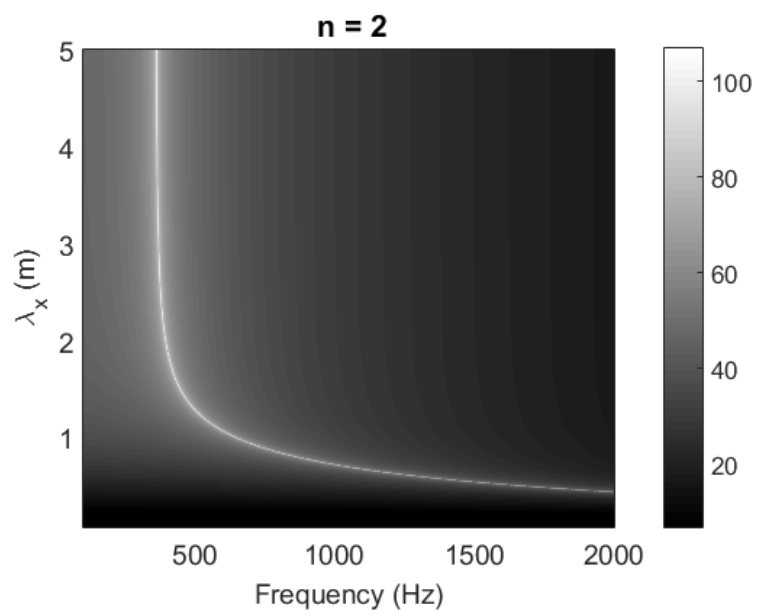

(c)

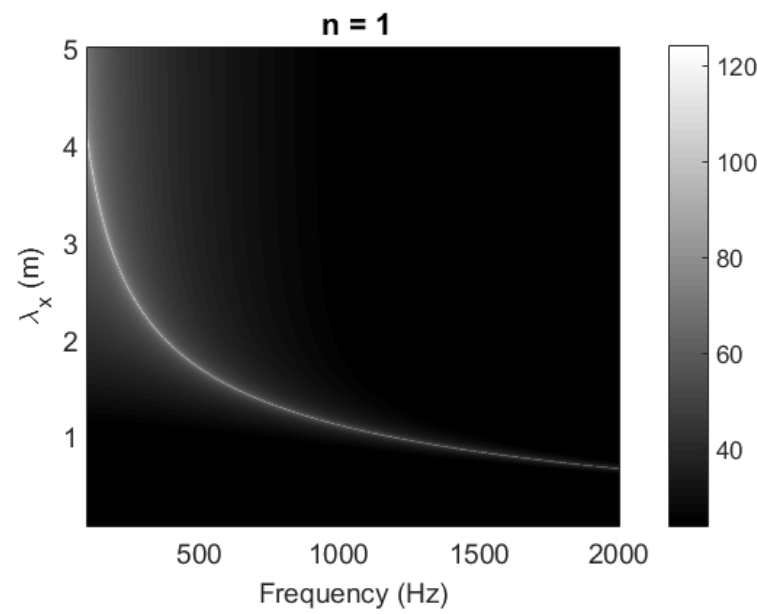

(b)

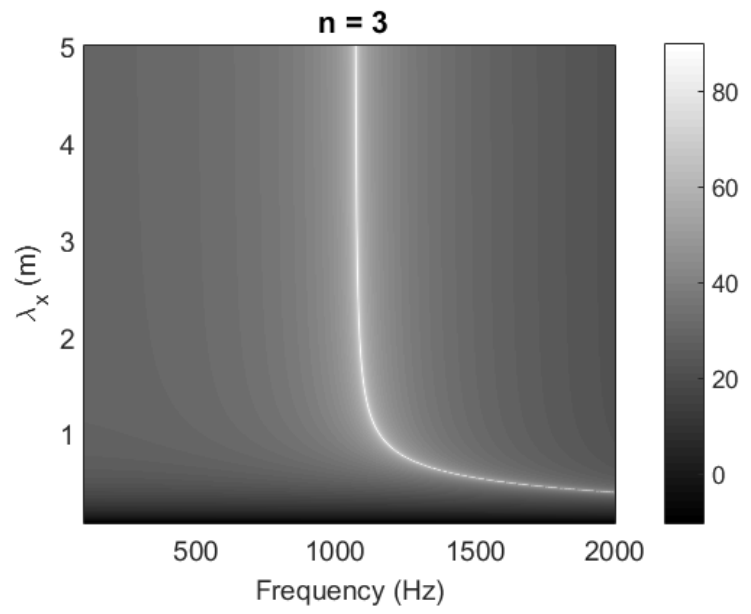

(d)

Figure 6. Level $\left(\mathrm{dB}\right.$, ref. $\left.1 \mathrm{~m}^{3}\right)$ of the spectral displacement of the fluid filled shell under a radial point force excitation as a function of frequency and axial wavelength $\lambda_{x}=2 \pi / k_{x}$ for:

(a) $n=0$. The dash, dash-dotted and continuous lines respectively denote the acoustic wavelength, the flexural wavelength (of an equivalent plate) and the longitudinal wavelength; (b) $n=1$ (c) $n=2$ (d) $n=3$. 


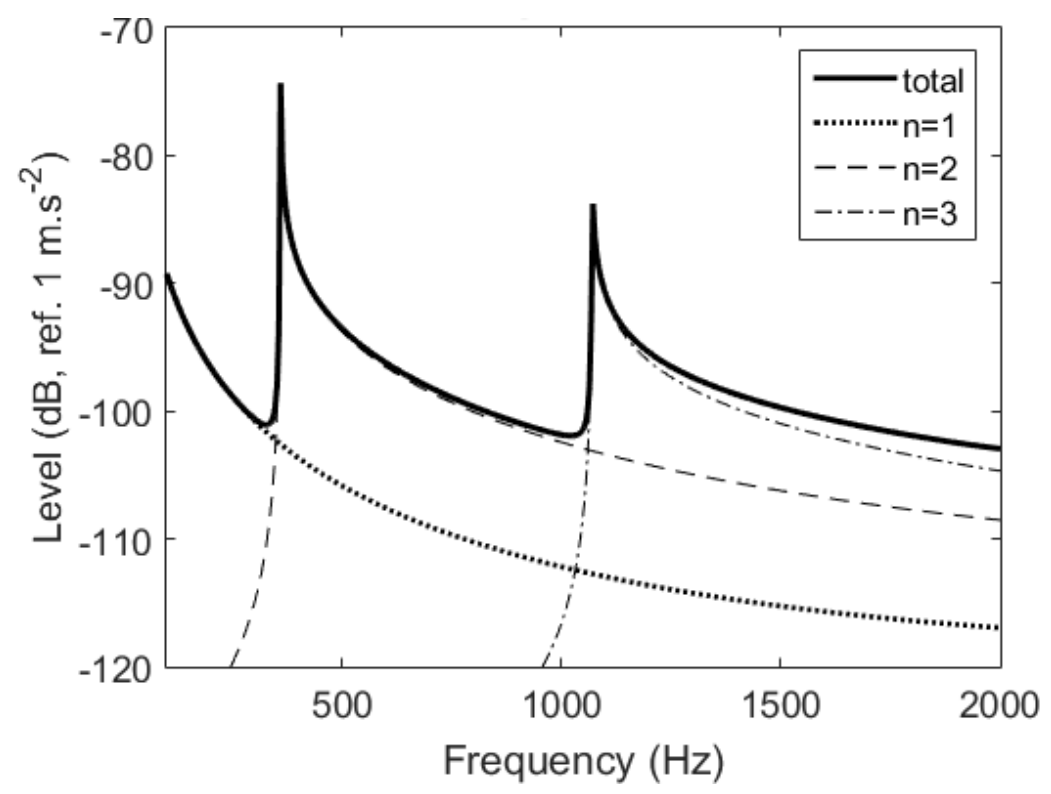

(a)

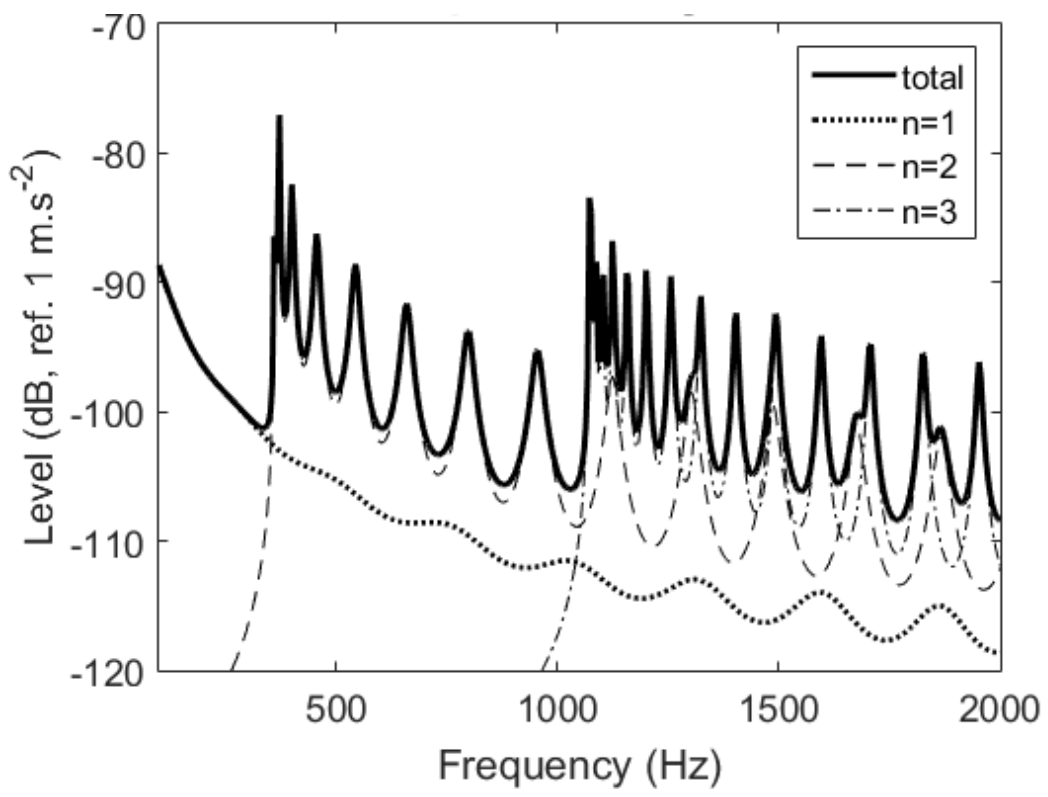

(b)

Figure 7. Mean ASD in $\mathrm{dB}$ (ref. $1 \mathrm{~ms}^{-2} \mathrm{~Hz}^{-1 / 2}$ ) of the accelerations measured by the virtual line array of 25 sensors. (a) shell without ring stiffeners, (b) shell with ring stiffeners. 


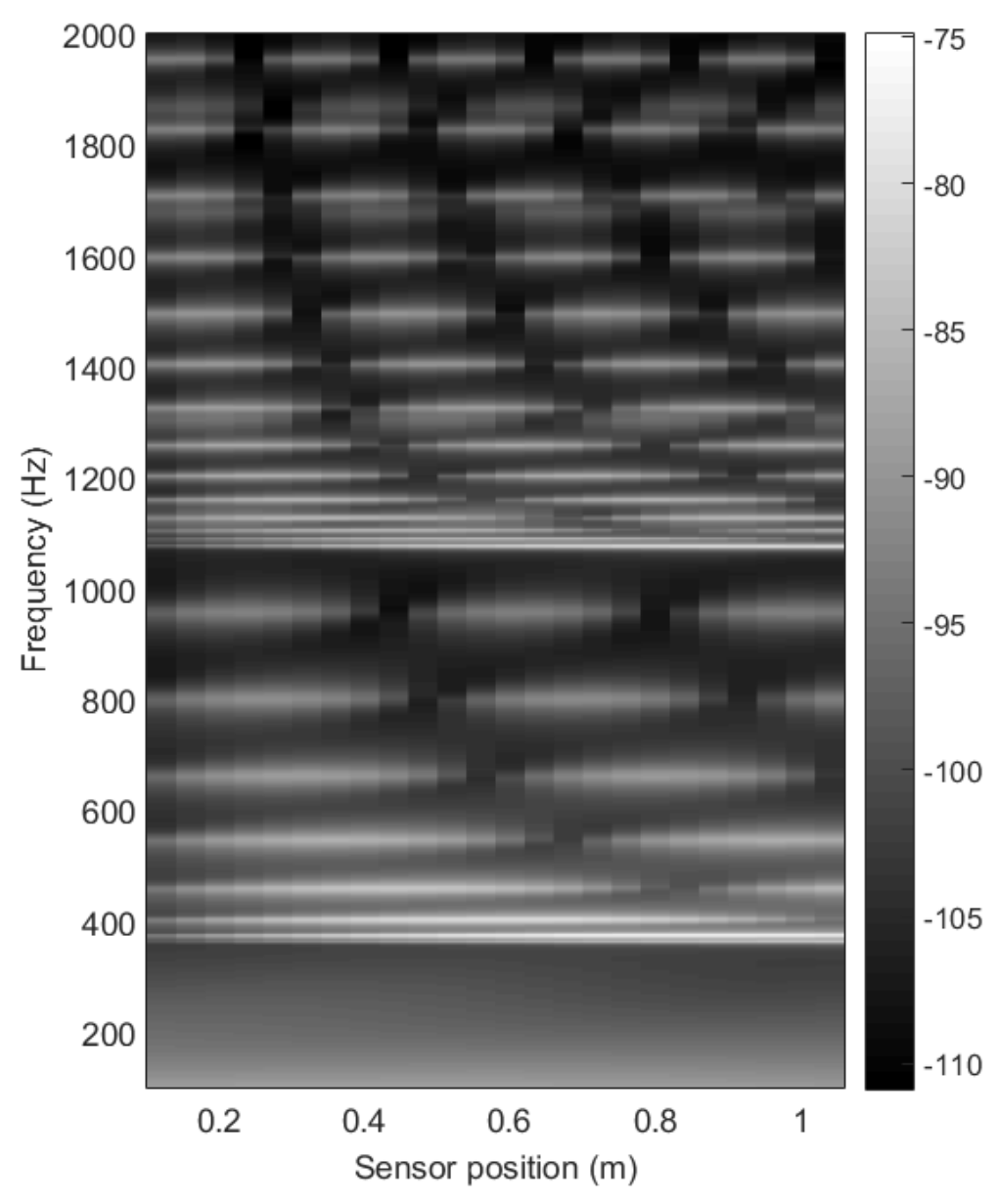

Figure 8. ASD of the accelerations in $\mathrm{dB}$ (ref. $1 \mathrm{~ms}^{-2} \mathrm{~Hz}^{-1 / 2}$ ) as a function of the sensor position and frequency, for the shell with ring stiffeners. 


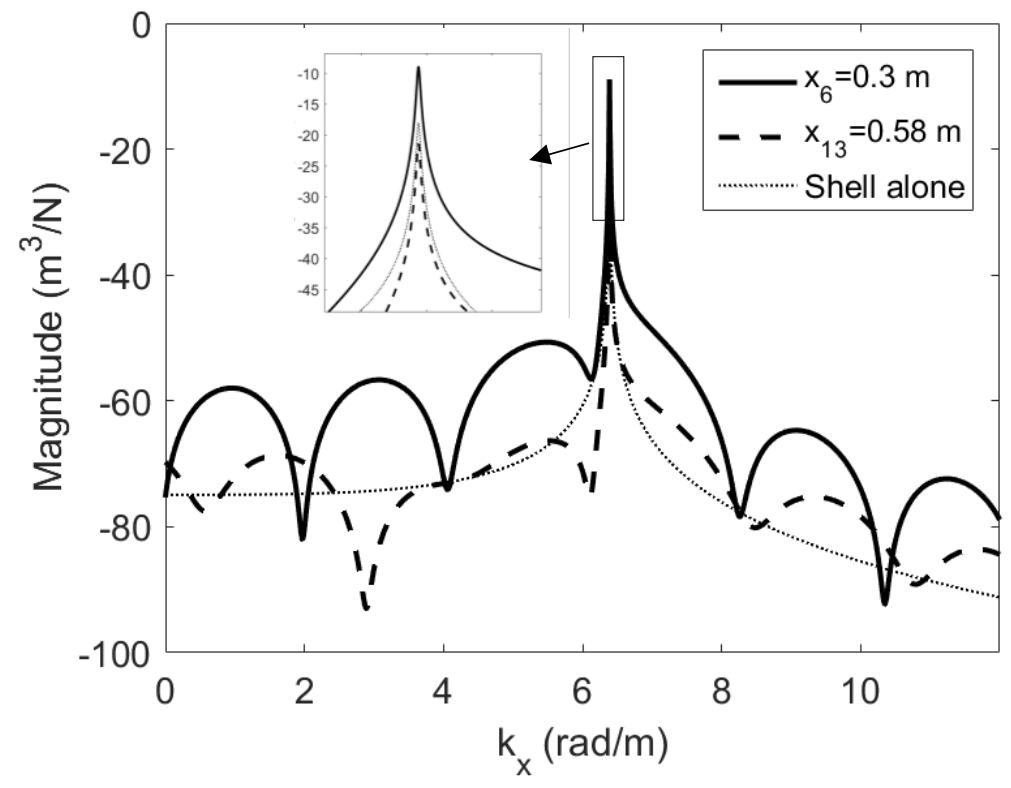

Figure 9. Magnitude of circumferential sensitivity functions, $\tilde{\tilde{H}}_{w}$ as a function of the positive axial wavenumber, $k_{x}$. Comparison for two response points on the shell coupled to the two ring stiffeners and for a point on the shell alone. Results at $660 \mathrm{~Hz}$ for $n=2$. 


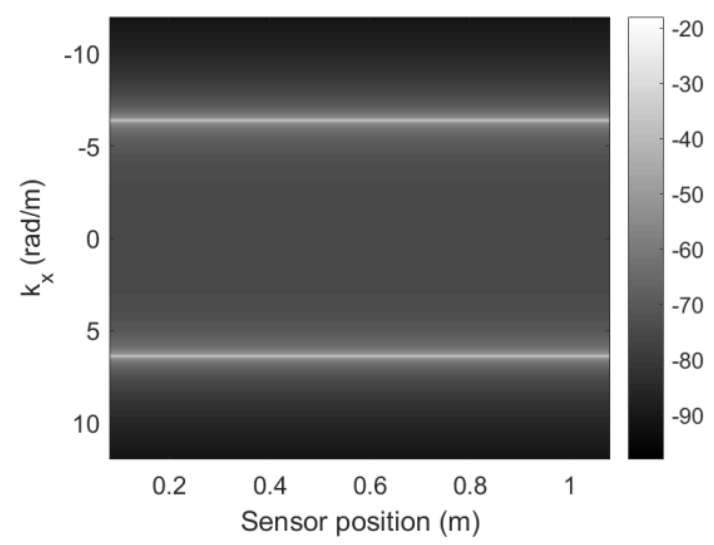

(a)

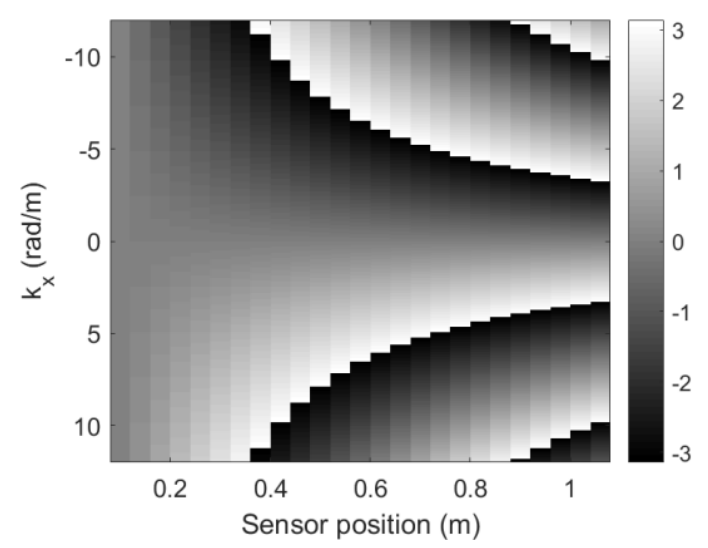

(c)

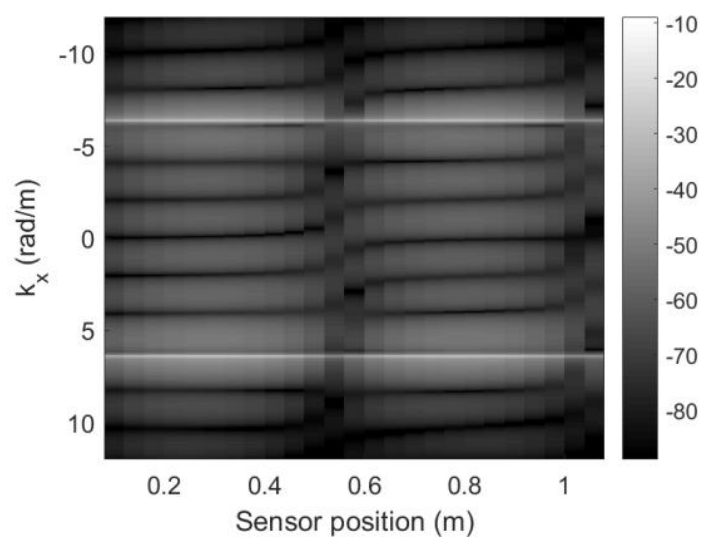

(b)

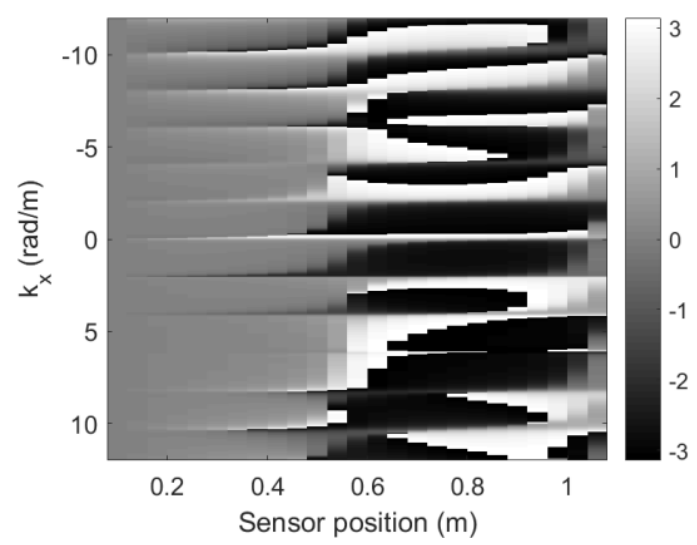

(d)

Figure 10. Comparison of circumferential sensitivity functions, $\tilde{\tilde{H}}_{w}$ as a function of the sensor position for the shell alone (a-c) and for the shell with the two ring stiffeners (b-d): (ab), magnitude (dB ref. $\left.1 \mathrm{Nm}^{3}\right)$; (c-d), phase difference between sensor \#1 and all the other ones (rad). Results at $660 \mathrm{~Hz}$ for $n=2$. 


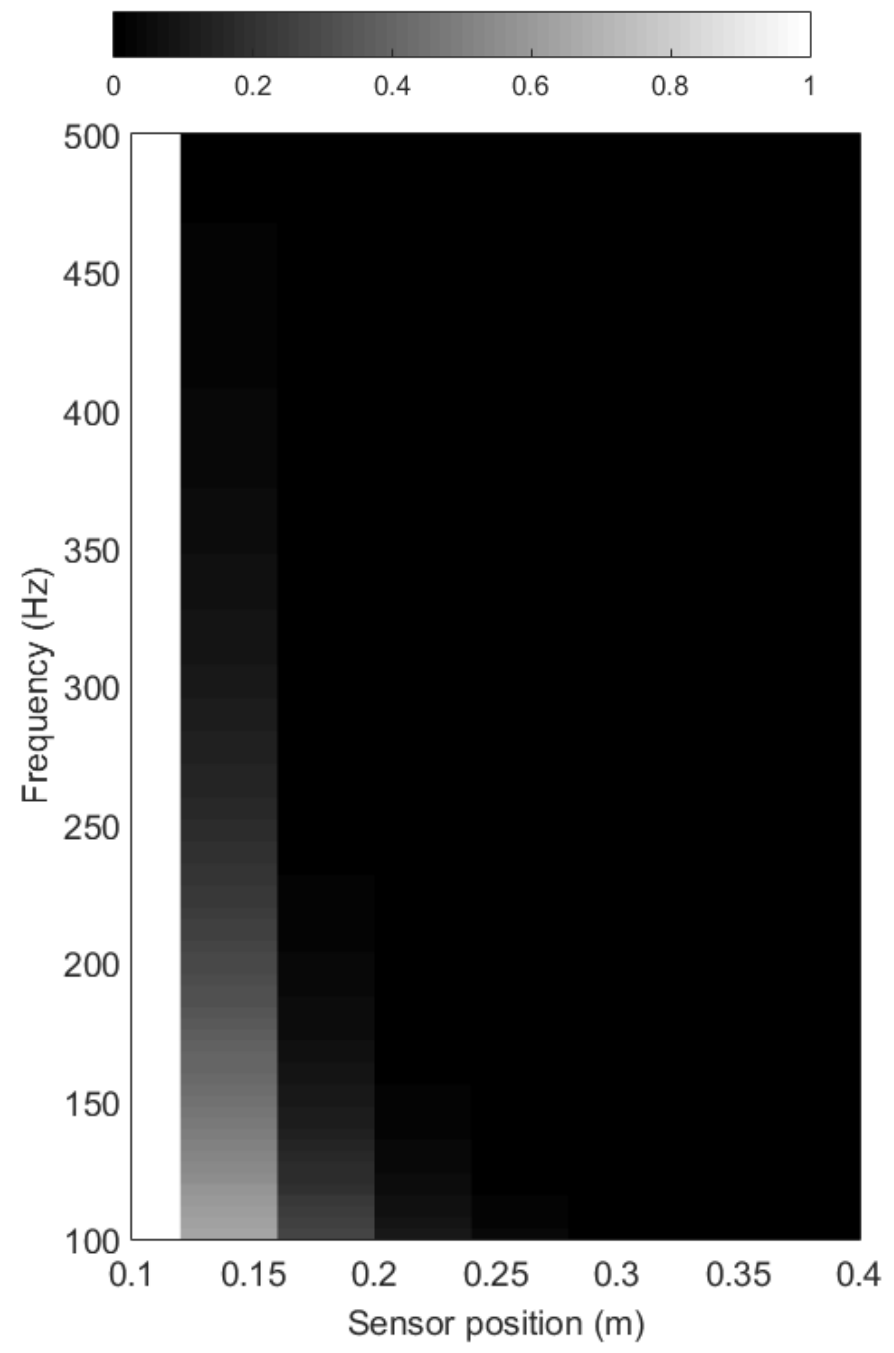

Figure 11. Normalized pressure CSD (i.e. coherence) between hydrophone \#1 and hydrophones \#2 to \#7, in a virtual hydrophone array with sensor spacing $0.04 \mathrm{~m}$. Numerical results obtained with Chase's 1987 model [13]. 
(a)

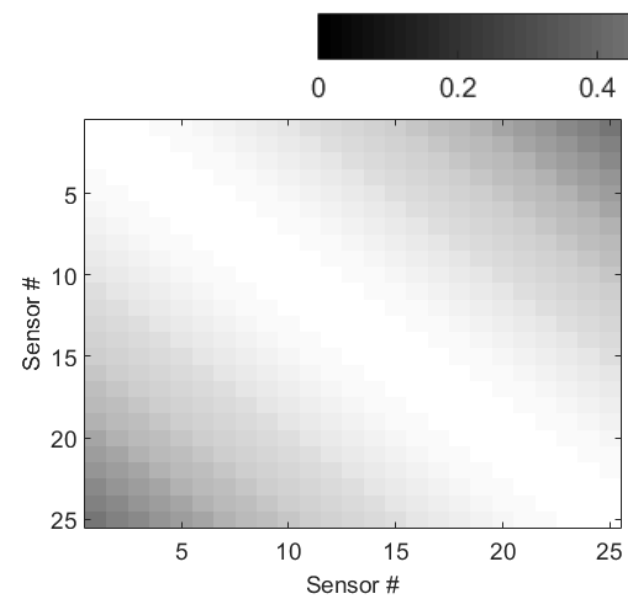

(b)

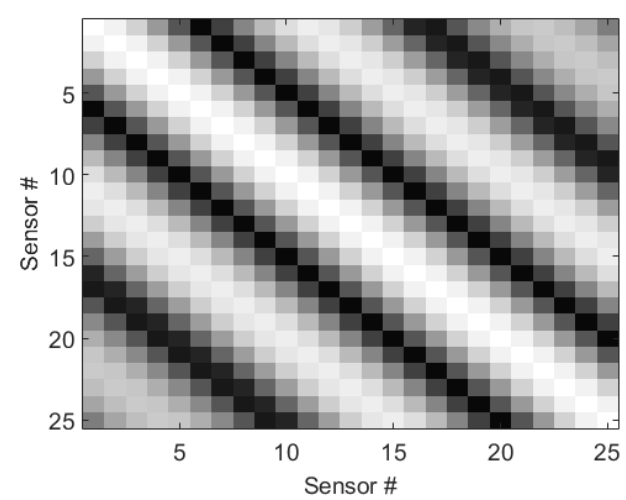

(c)

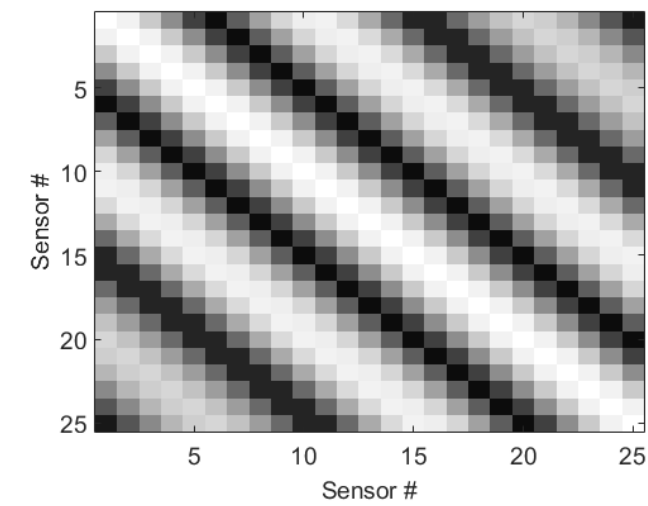

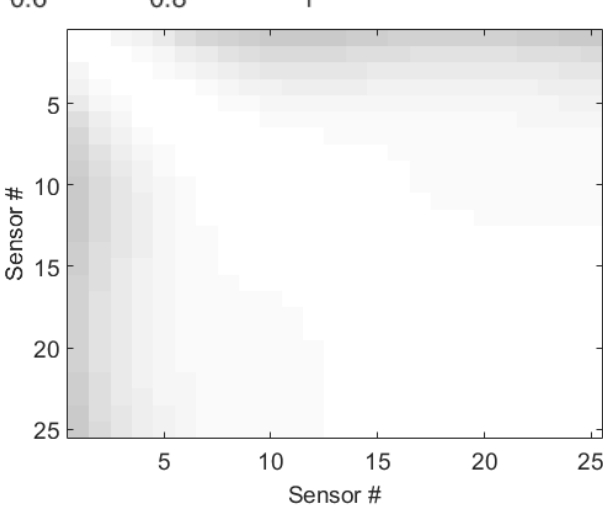

(d)

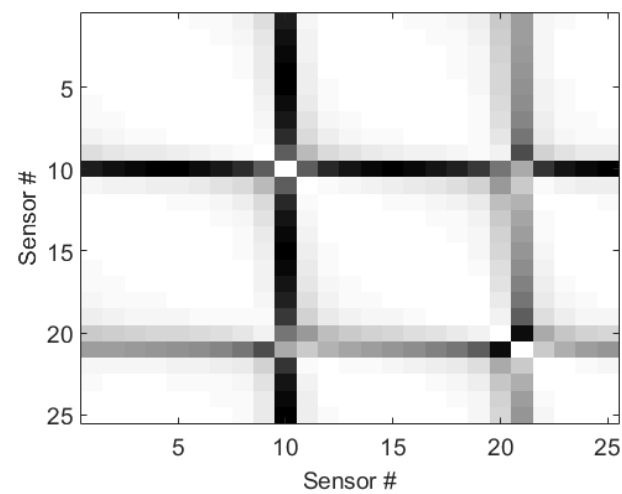

(e)

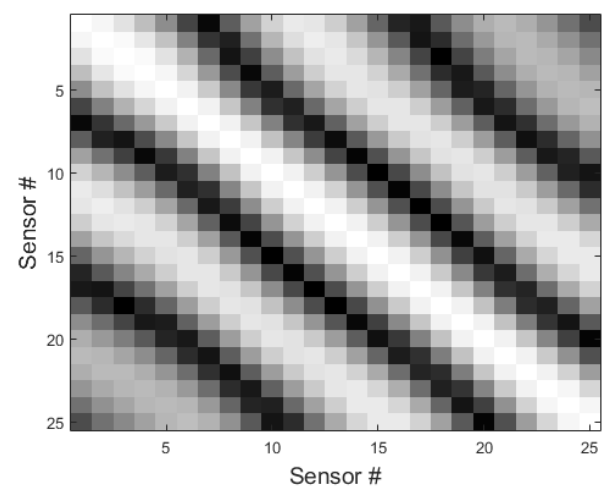

(f)

Figure 12. Normalized acceleration CSD between the 25 sensors. Two configurations: (a-c) shell without ring stiffeners and (d-f) shell with ring stiffeners. Three frequencies: (a,d) 1072 $\mathrm{Hz}$ (corresponding to the cut-on frequency $n=3$ ) (b,e) $1200 \mathrm{~Hz}$ (c,f) $1228 \mathrm{~Hz}$. 


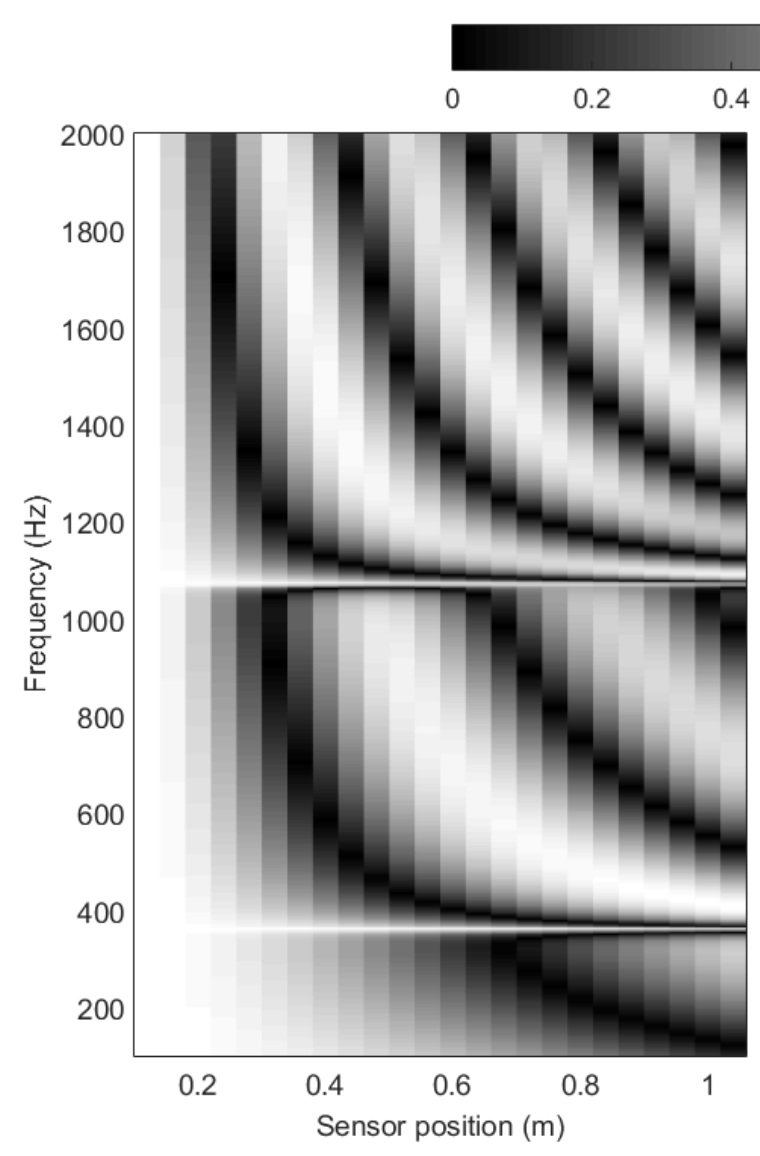

(a)

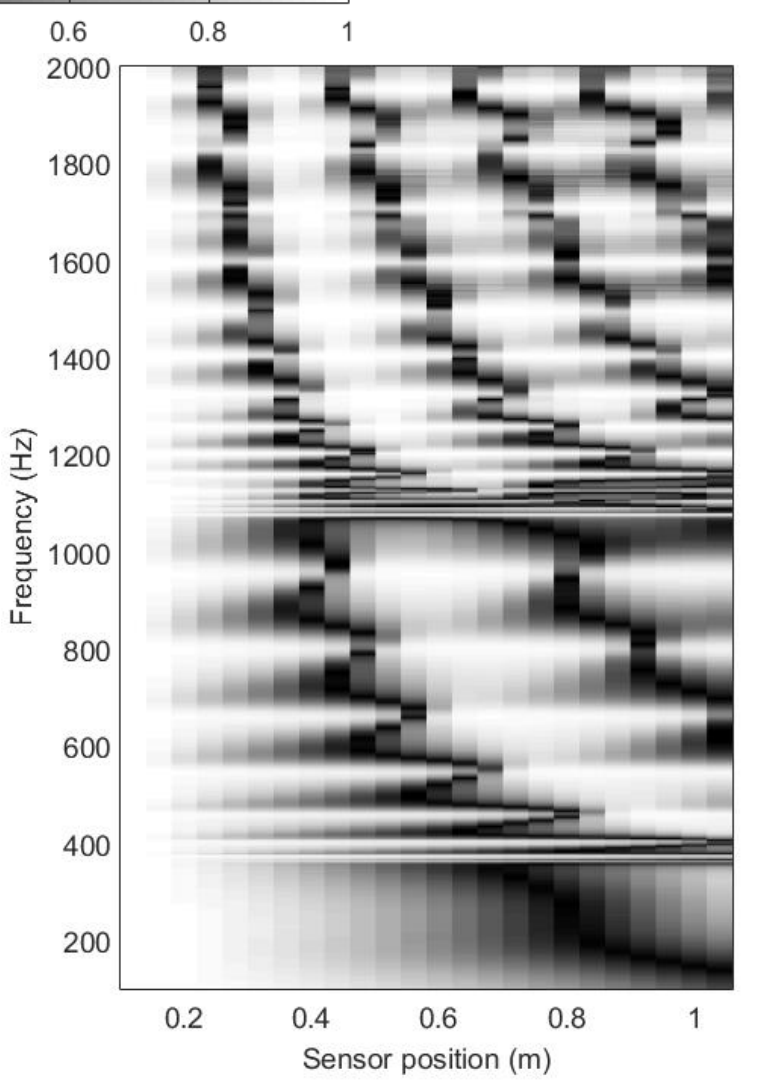

(b)

Figure 13. Normalized acceleration CSD between sensor \#1 and all the other ones as a function of sensor position and frequency. (a) shell without ring stiffeners (b) shell with two ring stiffeners. 


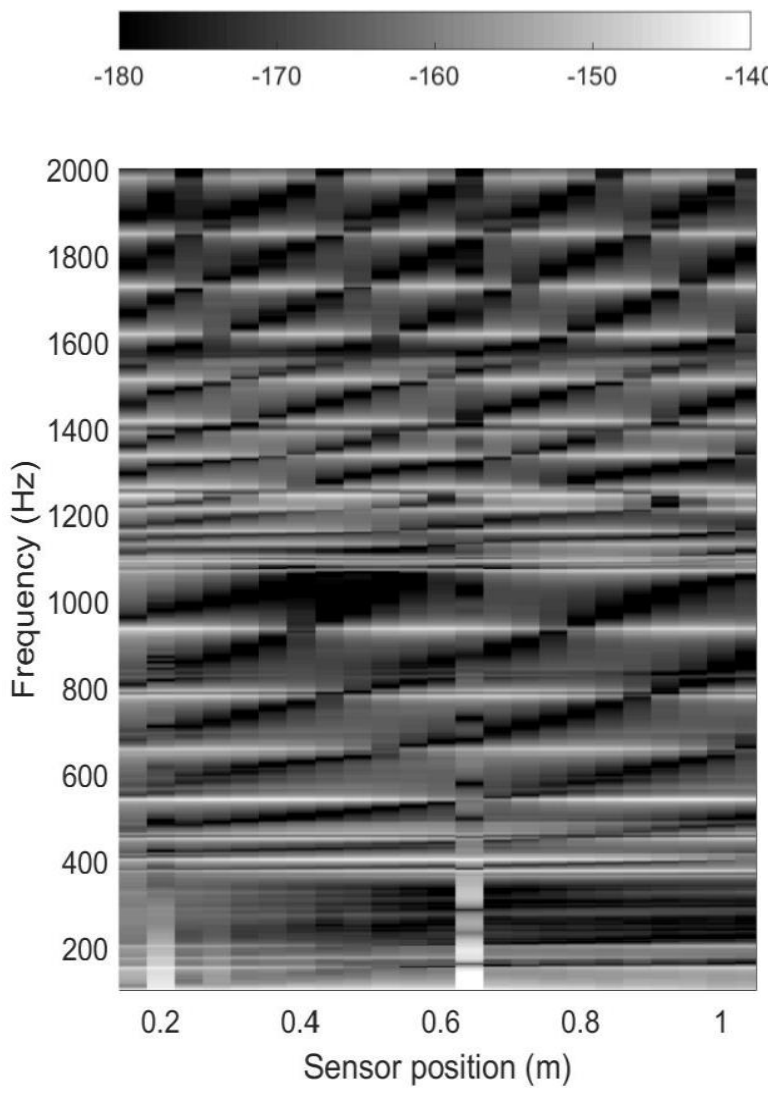

(a)

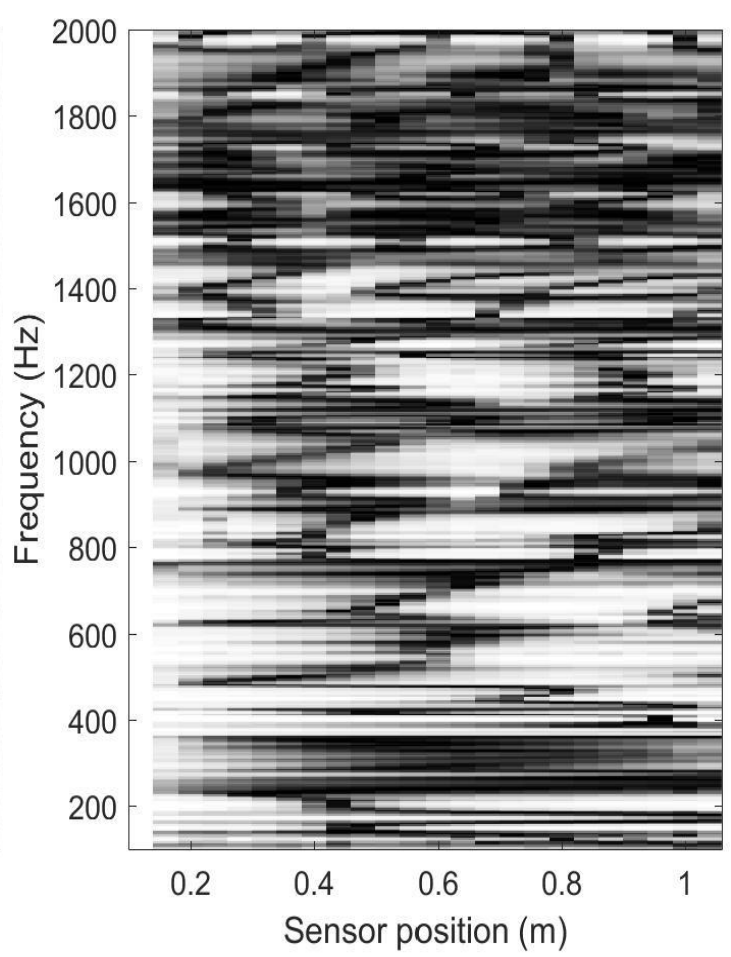

(b)

Figure 14. Experimental results on the laboratory mock-up [22]: (a) radial displacement response for a unit radial mechanical point force at $x=0.105 \mathrm{~m}$ (hammer excitation); (b) Normalized acceleration CSD between sensor \#1 and all the other sensors as a function of sensor position and frequency for a flow rate of $140 \mathrm{l} / \mathrm{s}$. 


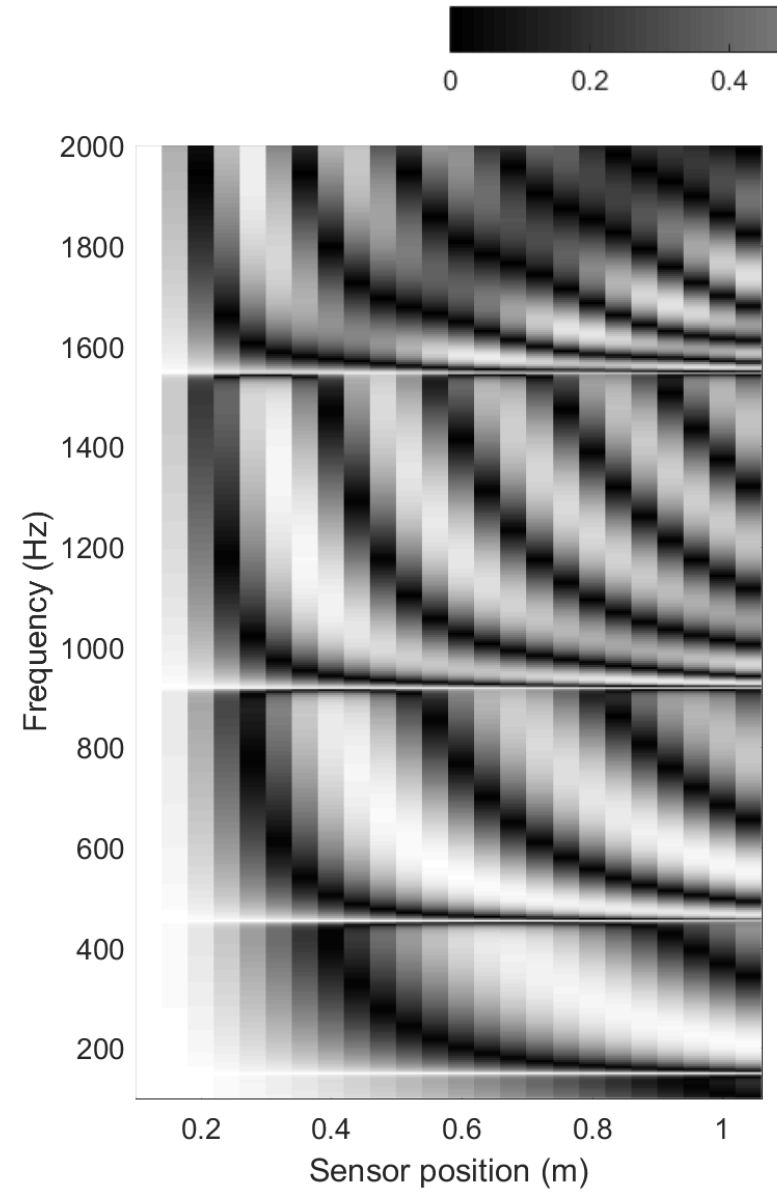

(a)

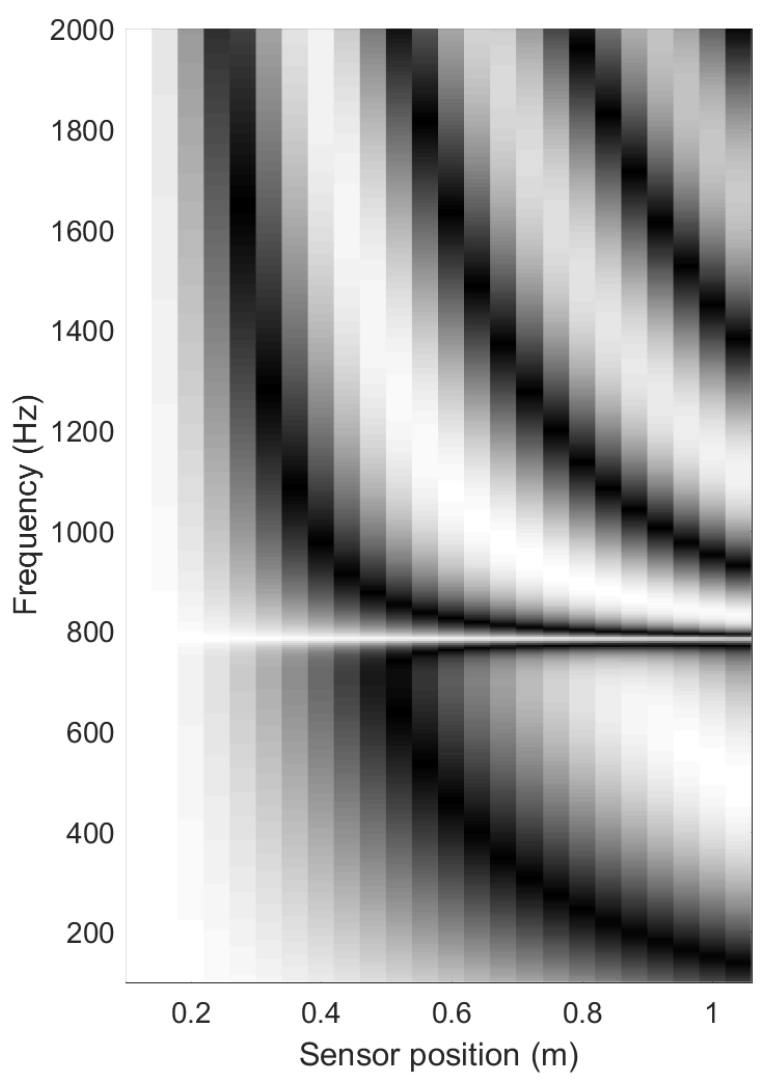

(b)

Figure 15. Normalized acceleration CSD between sensor \#1 and all the other sensors as a function of sensor position and frequency for the shell without ring stiffeners. Two different shell thicknesses: (a) $4 \mathrm{~mm}$ and (b) $16 \mathrm{~mm}$. 


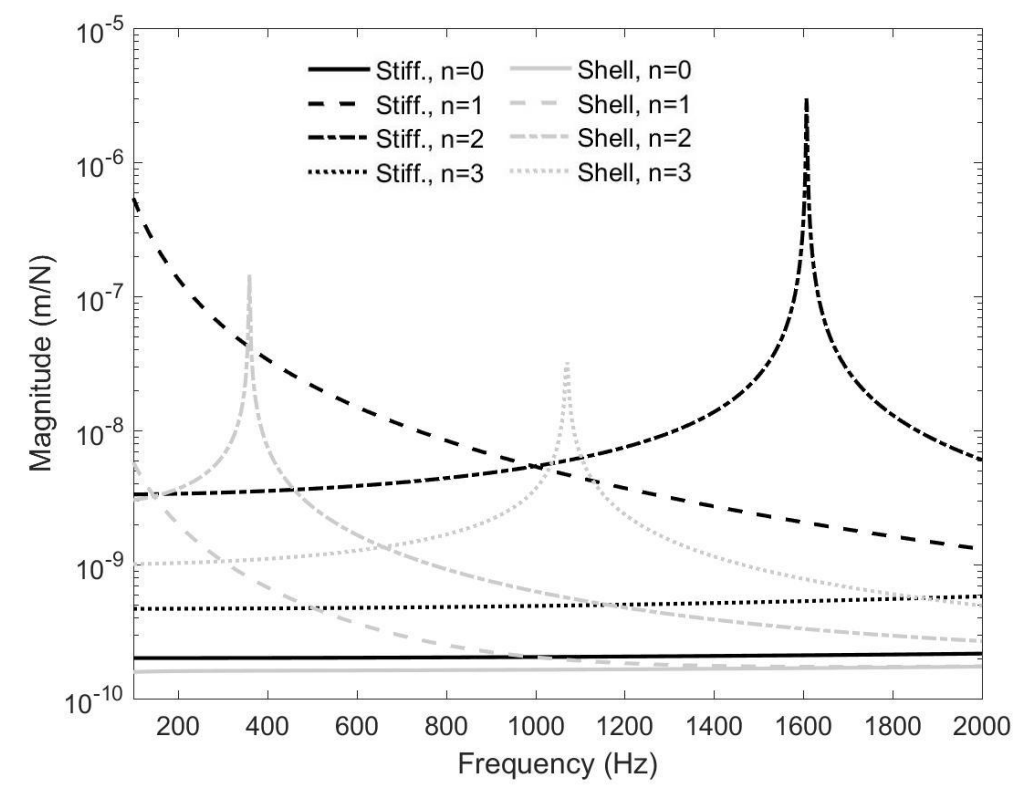

Figure 16. Amplitude of the circumferential admittances versus frequency for different circumferential orders. Comparison between the values for an $8 \mathrm{~mm}$ thick fluid filled cylindrical shell (named 'Shell' on the graph) and the values for a ring stiffener of $10 \times 30$ $\mathrm{mm}^{2}$ rectangular cross-section (named 'Stiff.' on the graph). 


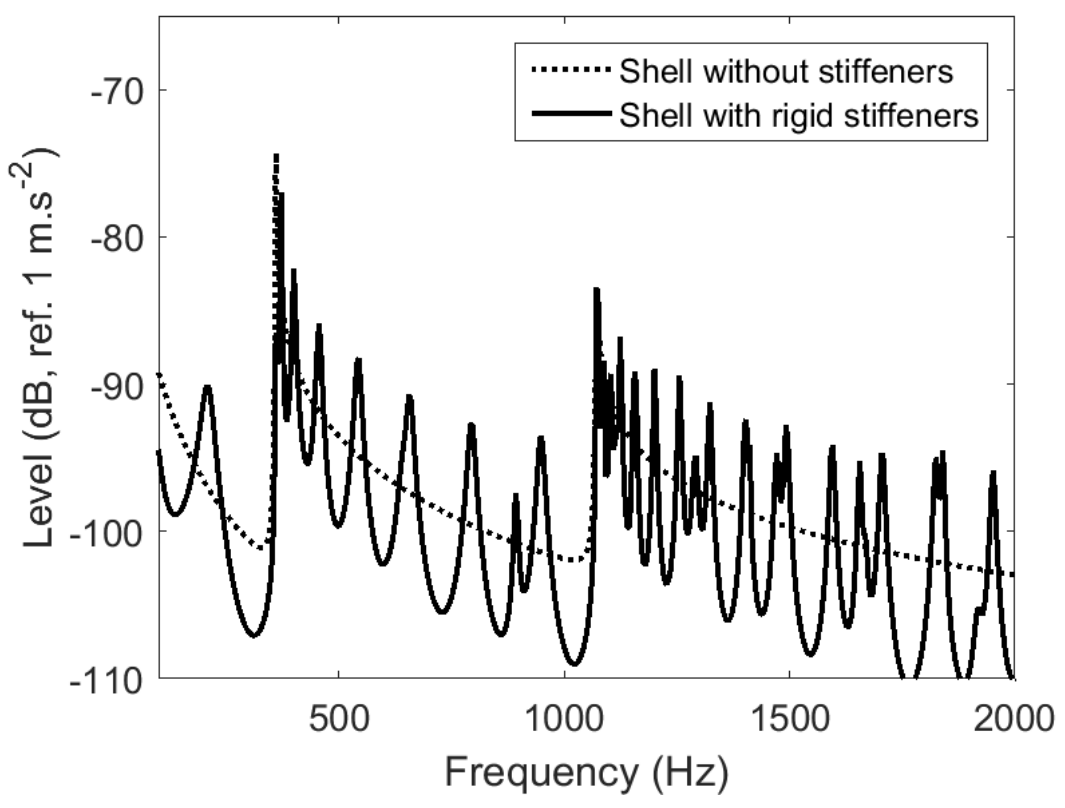

(a)

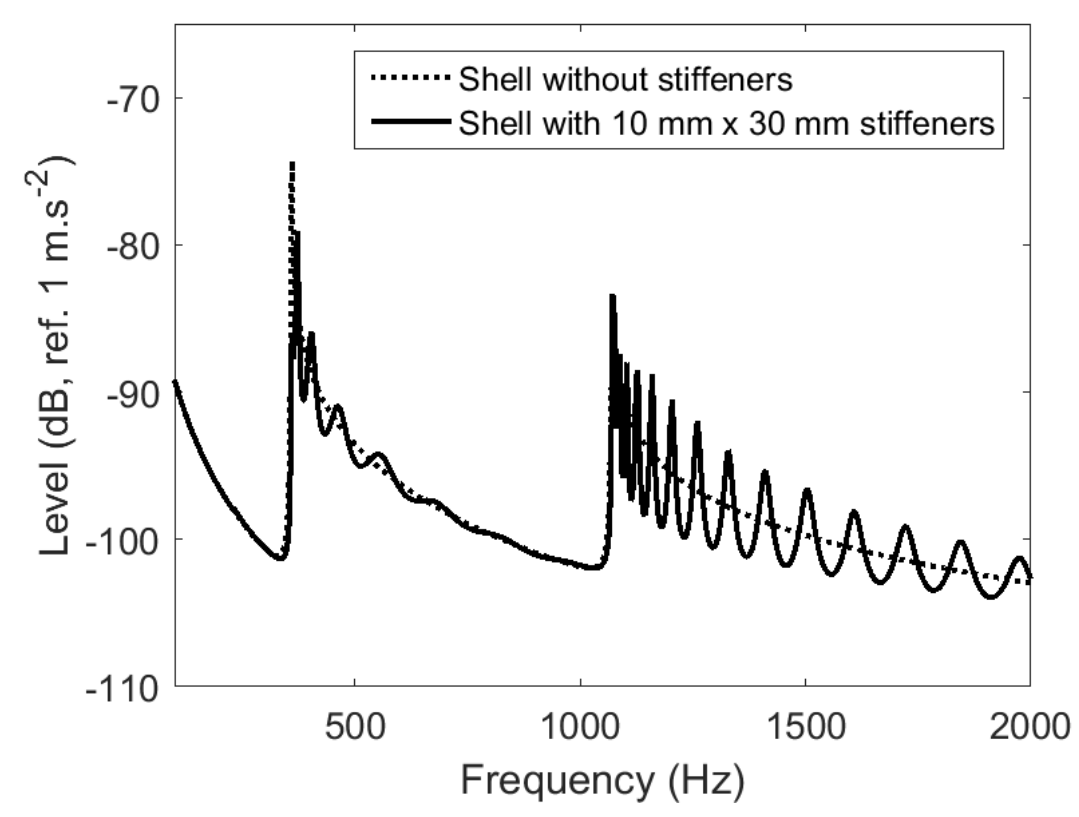

(b)

Figure 17. Mean ASD in $\mathrm{dB}$ (ref. $1 \mathrm{~ms}^{-2} \mathrm{~Hz}^{-1 / 2}$ ) of the accelerations measured by the virtual line array of 25 sensors. (a) shell with rigid ring stiffeners, (b) shell with ring stiffeners of 10 $\times 30 \mathrm{~mm}^{2}$ rectangular cross-section. 


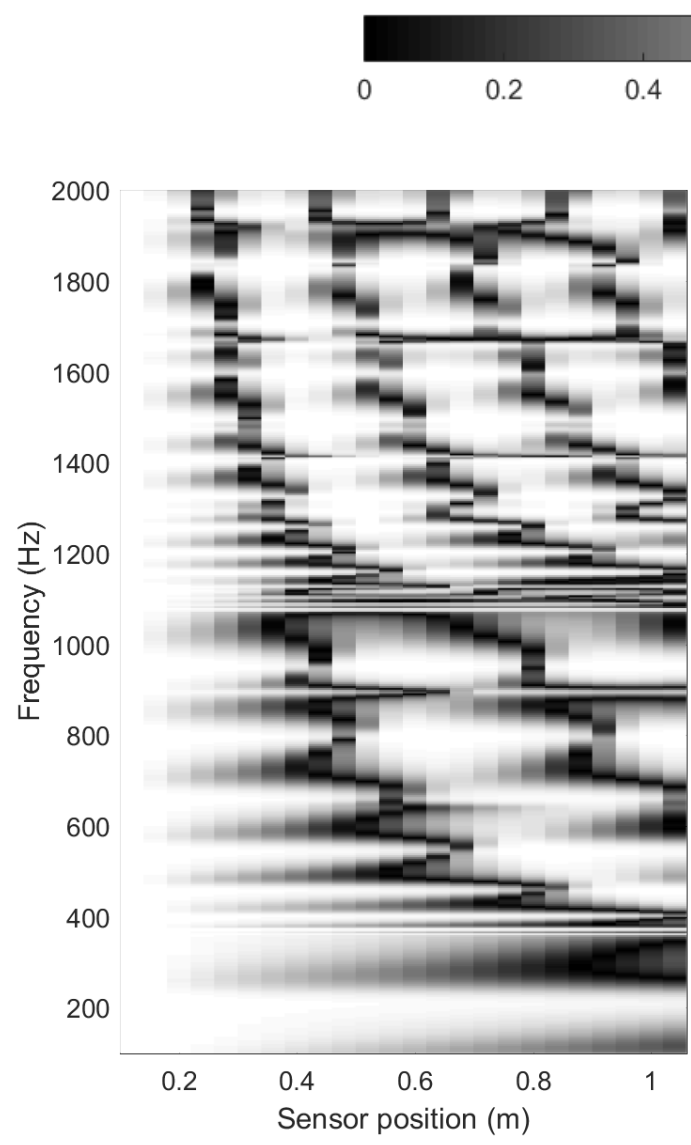

(a)

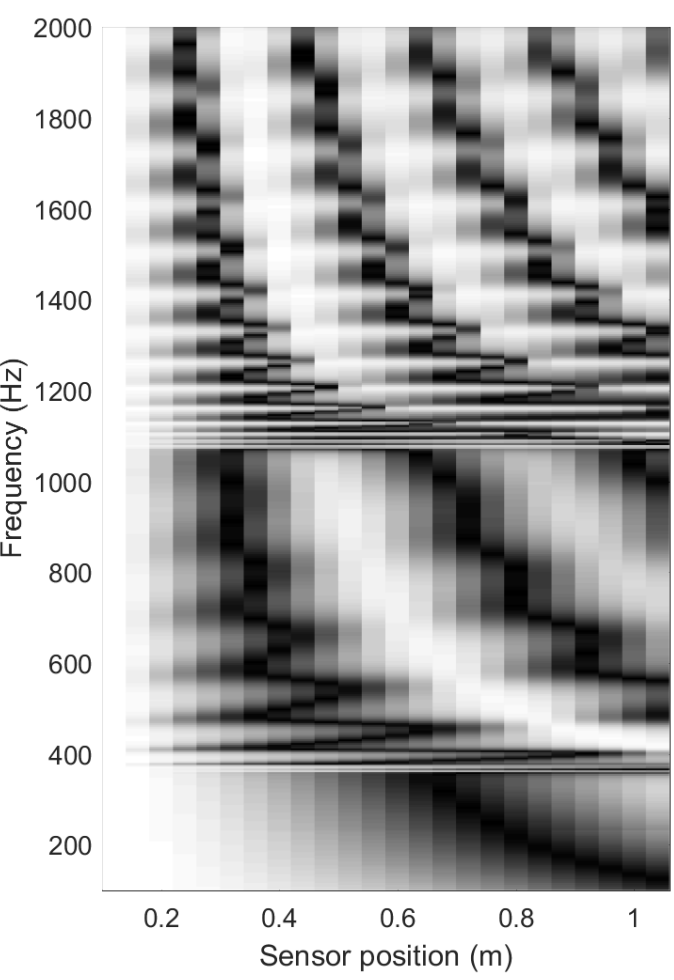

(b)

Figure 18. Normalized acceleration CSD between sensor \#1 and all the other sensors as a function of sensor position and frequency for the shell with two ring stiffeners; (a) totally rigid stiffeners, (b) stiffeners with a $10 \times 30 \mathrm{~mm}^{2}$ rectangular cross-section. 


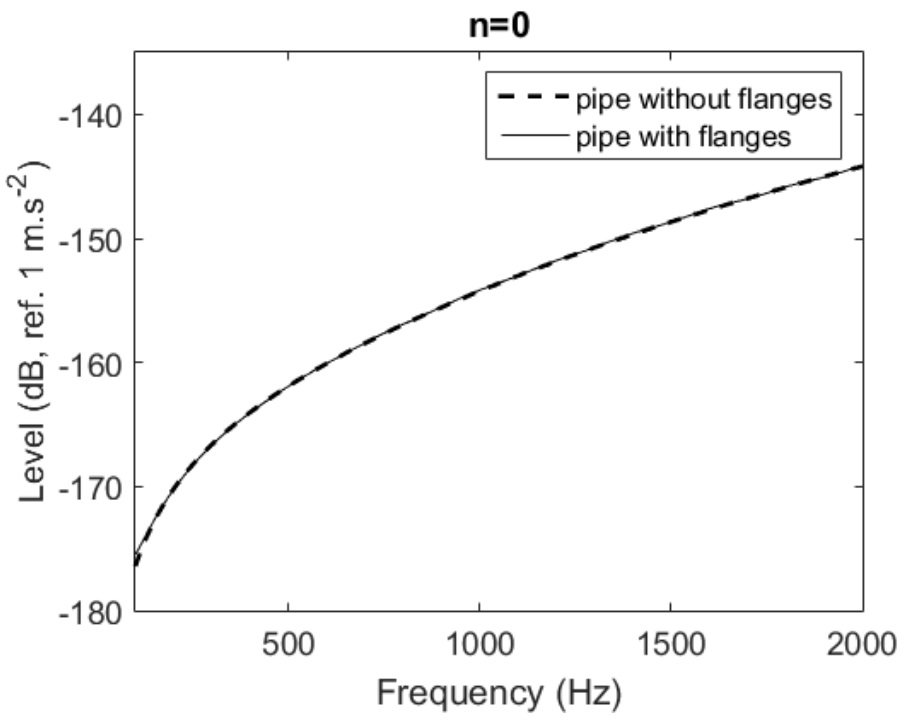

Figure 19. Mean value of the ASD of the accelerations in $\mathrm{dB}$ (ref. $1 \mathrm{~ms}^{-2} \mathrm{~Hz}^{-1 / 2}$ ) measured by the wire sensors. Measurements only include the contribution of the circumferential order $n=0$. The dashed line corresponds to the shell without stiffeners and the solid line to the shell with ring stiffeners. 


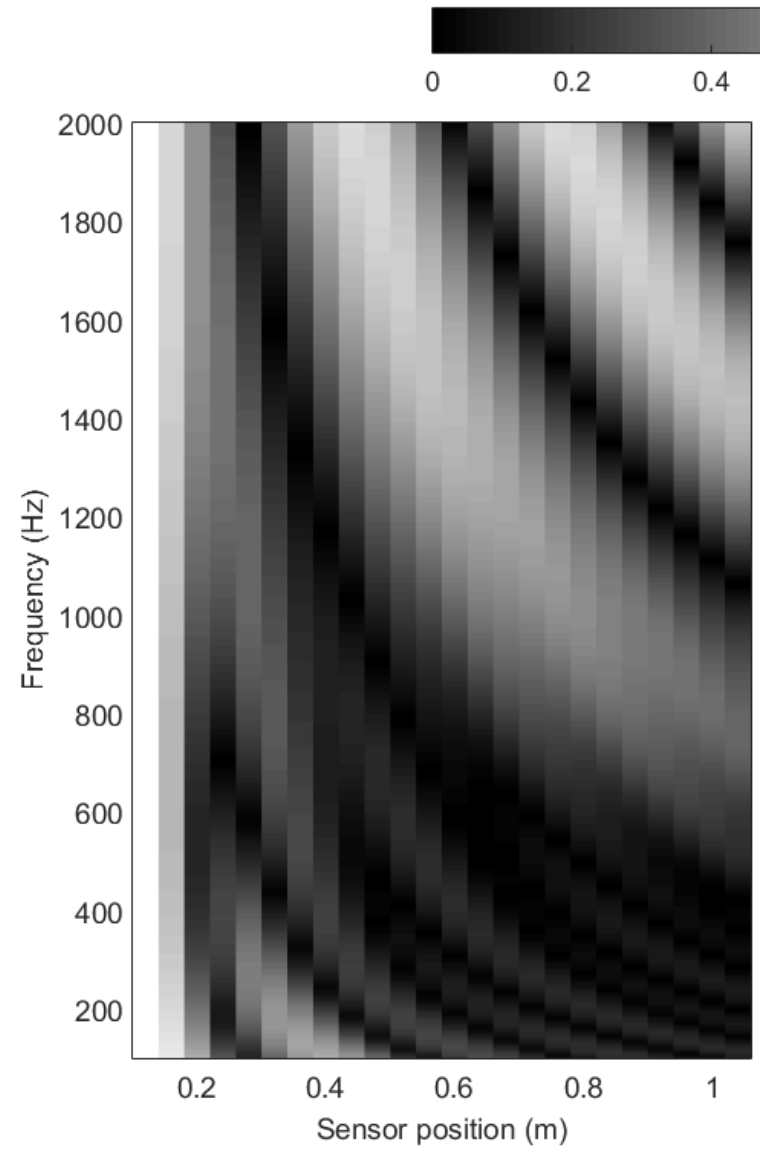

(a)

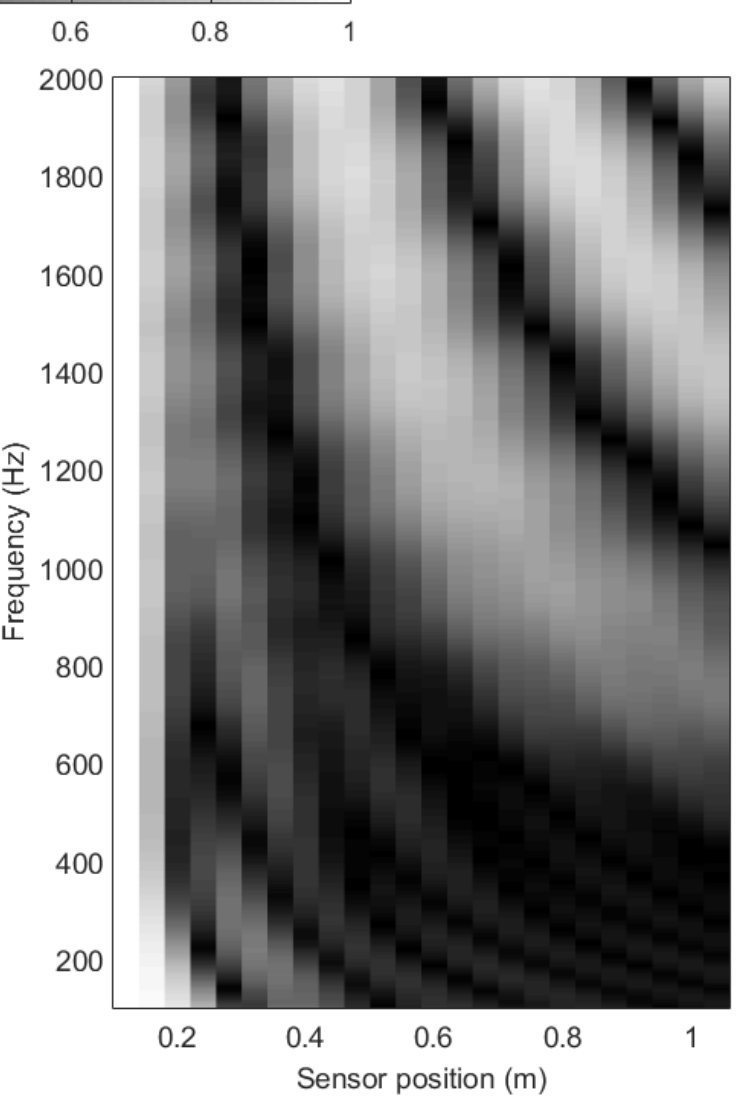

(b)

Figure 20. Normalized acceleration CSD between sensor \#1 and all other sensors as a function of sensor position and frequency using wire sensors. Contributions of the circumferential order $n=0$. (a) without stiffeners, (b) with stiffeners. 


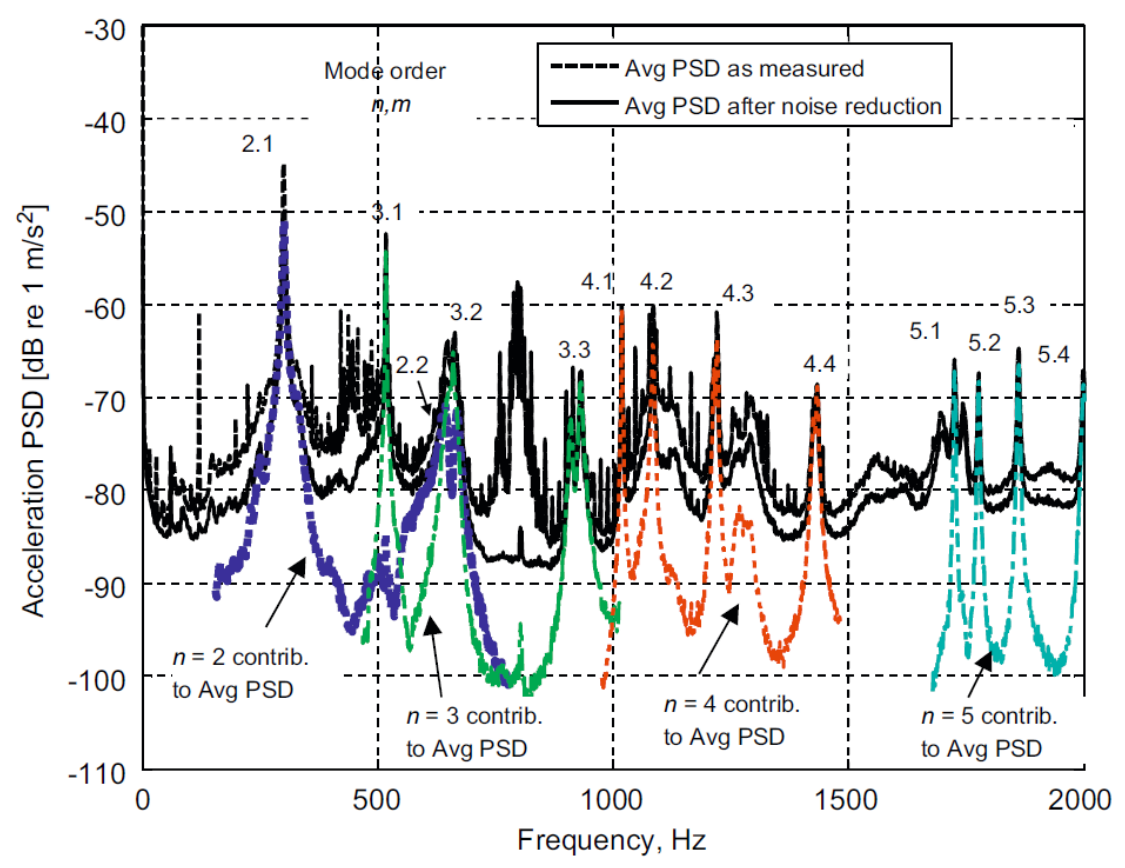

(a)

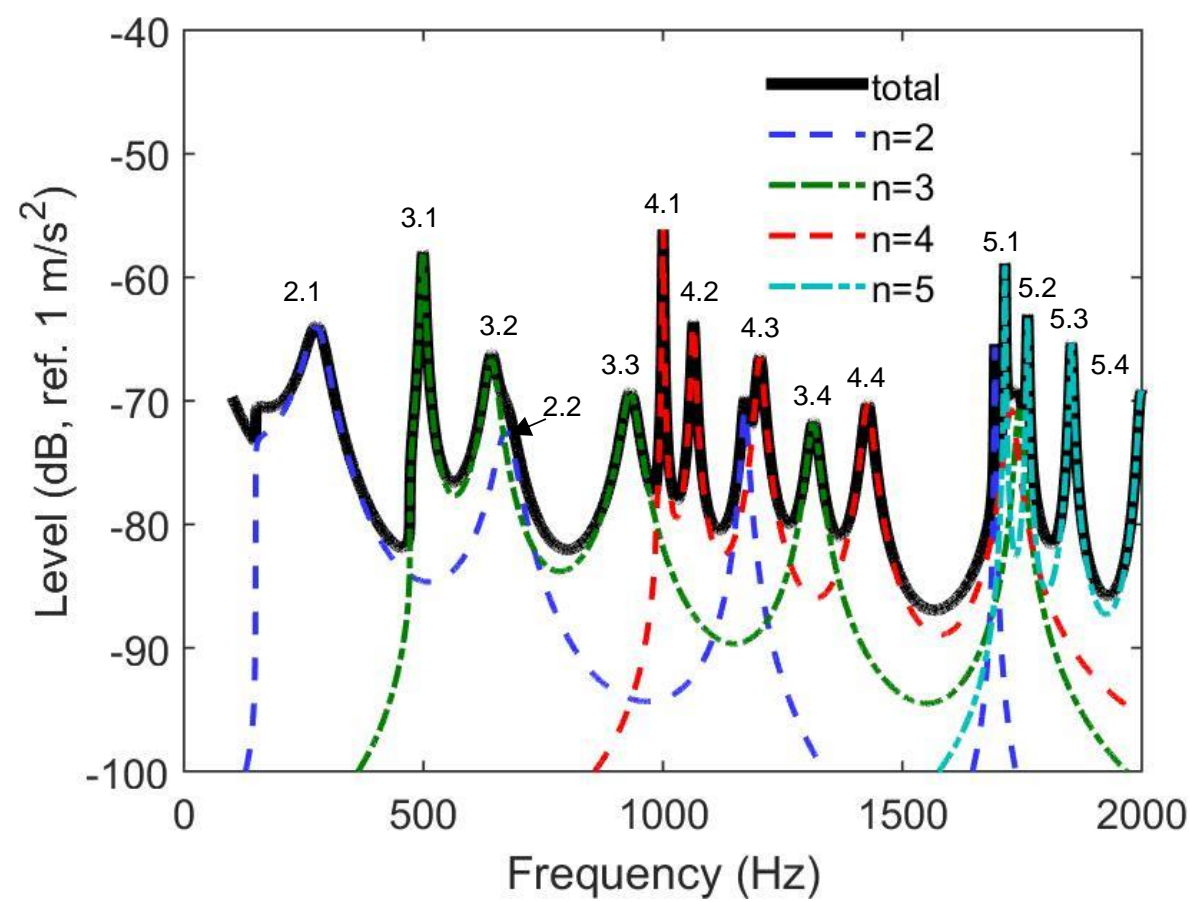

(b)

Figure B.1. Mean value of the ASD of the accelerations in $\mathrm{dB}$ (ref. $1 \mathrm{~ms}^{-2} \mathrm{~Hz}^{-1 / 2}$ ) measured by 3 rings of sensors. (a) experimental results of the Pennsylvania State University [33] (b) numerical results obtained with the proposed model. 